\title{
A quasilinear parabolic singular perturbation problem
}

\author{
Claudia Lederman ${ }^{\dagger}$ \\ Departamento de Matemática, Facultad de Ciencias Exactas y Naturales, \\ Universidad de Buenos Aires, 1428 Buenos Aires, Argentina \\ Dietmar OelZ \\ Faculty of Mathematics, Vienna University, Nordbergstr. 15, A 1090 Wien, Austria
}

[Received 17 February 2007 and in revised form 16 July 2008]

We study the following singular perturbation problem for a quasilinear uniformly parabolic operator of interest in combustion theory:

$$
\operatorname{div} F\left(\nabla u^{\varepsilon}\right)-\partial_{t} u^{\varepsilon}=\beta_{\varepsilon}\left(u^{\varepsilon}\right),
$$

where $u^{\varepsilon} \geqslant 0, \beta_{\varepsilon}(s)=(1 / \varepsilon) \beta(s / \varepsilon), \varepsilon>0, \beta$ is Lipschitz continuous, $\operatorname{supp} \beta=[0,1]$ and $\beta>0$ in $(0,1)$. We obtain uniform estimates, we pass to the limit $(\varepsilon \rightarrow 0)$ and we show that, under suitable assumptions, the limit function $u$ is a solution to the free boundary problem

$$
\begin{array}{ll}
\operatorname{div} F(\nabla u)-\partial_{t} u=0 & \text { in }\{u>0\}, \\
u_{v}=\alpha(v, M) & \text { on } \partial\{u>0\},
\end{array}
$$

in a pointwise sense and in a viscosity sense. Here $u_{v}$ denotes the derivative of $u$ with respect to the inward unit spatial normal $v$ to the free boundary $\partial\{u>0\}, M=\int \beta(s) \mathrm{d} s, \alpha(v, M):=\Phi_{v}^{-1}(M)$ and $\Phi_{\nu}(\alpha):=-A(\alpha \nu)+\alpha \nu \cdot F(\alpha \nu)$, where $A(p)$ is such that $F(p)=\nabla A(p)$ with $A(0)=0$. Some of the results obtained are new even when the operator under consideration is linear.

2000 Mathematics Subject Classification: Primary 35R35, 35K55; Secondary 80A25.

Keywords: Quasilinear parabolic operator; singular perturbation problem; free boundary problem; combustion.

\section{Introduction}

In this paper we study a singular perturbation problem for a quasilinear uniformly parabolic operator of interest in combustion theory. The problem under consideration is the following:

$$
\operatorname{div} F\left(\nabla u^{\varepsilon}\right)-\partial_{t} u^{\varepsilon}=\beta_{\varepsilon}\left(u^{\varepsilon}\right),
$$

in a domain $\mathcal{D} \subseteq \mathbb{R}^{N+1}$ with $u^{\varepsilon} \geqslant 0, \beta_{\varepsilon}(s):=(1 / \varepsilon) \beta(s / \varepsilon)$ and $\varepsilon>0$ small. Here $\beta$ is a Lipschitz continuous function, $\operatorname{supp} \beta=[0,1]$ and $\beta>0$ in $(0,1)$.

In the particular case of the heat operator, problem $\left(P_{\varepsilon}\right)$ takes the form

$$
\Delta u^{\varepsilon}-\partial_{t} u^{\varepsilon}=\beta_{\varepsilon}\left(u^{\varepsilon}\right) .
$$

\footnotetext{
†E-mail: clederma@dm.uba.ar

E-mail: dietmar.oelz@univie.ac.at
} 
This problem appears in combustion theory in the description of the propagation of curved, premixed deflagration flames. The function $u^{\varepsilon}$ represents the normalized temperature of the mixture, more precisely, $u^{\varepsilon}=\lambda\left(T_{f}-T^{\varepsilon}\right)$ where $T_{f}$ is the flame temperature and $\lambda$ is a normalization factor. We point out that $u^{\varepsilon}$ may also be seen as the concentration of the reactant, so it is in general assumed to be nonnegative. The parameter $\varepsilon$ involved in the model represents the inverse of the activation energy (see Appendix $\mathrm{B}$ for the derivation of equations $(1.1)$ and $\left(P_{\varepsilon}\right)$ ).

The study of the limit as $\varepsilon \rightarrow 0$ (the high activation energy analysis) of problem (1.1) was proposed in the 1930s (see [29]) and has received a lot of attention in recent years. It has been shown that, under certain assumptions, it is possible to pass to the limit in (1.1) and that the limit function $u$ is a solution to the free boundary problem

$$
\begin{array}{ll}
\Delta u-\partial_{t} u=0 & \text { in }\{u>0\}, \\
|\nabla u|=\sqrt{2 M} & \text { on } \partial\{u>0\},
\end{array}
$$

where $M=\int \beta(s) \mathrm{d} s$. We refer to [5, 17, 13, 14, 15, 23, 18, 28, 24, 1] for the precise results and assumptions. See also the survey paper [27].

In the present study we consider a family of nonnegative solutions to problem $\left(P_{\varepsilon}\right)$ and we show that under suitable assumptions the limit function $u$ is a solution to the free boundary problem

$$
\begin{array}{ll}
\operatorname{div} F(\nabla u)-\partial_{t} u=0 & \text { in }\{u>0\}, \\
\Phi(\nabla u)=M & \text { on } \partial\{u>0\},
\end{array}
$$

in a sense that will be made precise later. Here $M$ is as above, $\Phi(p):=-A(p)+p \cdot F(p)$ and $A(p)$ is such that $F(p)=\nabla A(p)$ with $A(0)=0$.

More precisely, we show that under suitable assumptions the limit function $u$ is a solution to the free boundary problem

$$
\begin{array}{ll}
\operatorname{div} F(\nabla u)-\partial_{t} u=0 & \text { in }\{u>0\}, \\
u_{v}=\alpha(\nu, M) & \text { on } \partial\{u>0\},
\end{array}
$$

where $u_{v}$ denotes the derivative of $u$ with respect to the inward unit spatial normal $v$ to the free boundary $\partial\{u>0\}, \alpha(\nu, M):=\Phi_{\nu}^{-1}(M)$ and $\Phi_{\nu}(\alpha):=-A(\alpha \nu)+\alpha \nu \cdot F(\alpha \nu)(M$ and $A$ as above).

We point out that the derivation of the functions $\Phi$ and $\Phi_{v}$ appearing in the free boundary condition requires a nontrivial analysis. Therefore we postpone it to Section 5

Let us observe that we may also write the free boundary condition as

$$
|\nabla u|=\alpha(\nu, M) \quad \text { on } \partial\{u>0\},
$$

with $\nu, M$ and $\alpha(\nu, M)$ as before.

In order to achieve our goal we proceed as follows: in Section 4 we consider a family $u^{\varepsilon}$ of nonnegative solutions to $\left(P_{\varepsilon}\right)$ in a domain $\mathcal{D} \subset \mathbb{R}^{N+1}$ which are uniformly bounded in $L^{\infty}$ norm in $\mathcal{D}$ and we obtain uniform estimates on the family $u^{\varepsilon}$ that allow the passage to the limit as $\varepsilon \rightarrow 0$. We also show that the limit function $u$ satisfies

$$
\operatorname{div} F(\nabla u)-\partial_{t} u=\mu
$$

with $\mu$ a nonnegative Radon measure supported on the free boundary $\mathcal{D} \cap \partial\{u>0\}$. 
In Section 5 we analyze some particular limits that are crucial to understanding the behavior of general limits. In particular, we show that the free boundary condition is satisfied in the special case the limit function is $u=\alpha\left\langle x-x_{0}, v\right\rangle^{+}$for some unit vector $v \in \mathbb{R}^{N}$ and $\alpha>0$ (Theorem 5.1. We remark that obtaining such a result in the evolutionary case is a nontrivial matter-as occurred with the heat operator (cf. [14, 28, 24]). Thus, delicate auxiliary estimates are required to achieve this purpose (see Lemma 4.5). In Section 5 we also present some examples of different operators and we exhibit the resulting free boundary condition.

In Section 6 we prove upper bounds for the gradient of limit functions, which are related to the free boundary condition.

In Section 7 we show that, under suitable assumptions, the free boundary condition is satisfied in a pointwise sense. This is so either at free boundary points where there is an inward spatial normal in the parabolic measure-theoretic sense (Theorem[7.1), or at free boundary points where the free boundary is locally a differentiable surface (Theorem 7.2 , provided a nondegeneracy condition holds at that point.

Finally, we prove in Section 8 (Theorems 8.1 and 8.2 that limit functions are viscosity supersolutions and viscosity subsolutions of the free boundary problem $(P)$ under certain assumptions (see Remarks 8.2 to 8.4 for a discussion of our assumptions).

Let us mention that Theorem 8.2 is new even if the operator is linear, since the results of this kind available in the literature for the heat operator in the one phase case are obtained either under stronger assumptions on the limit function $u$ or with a different concept of viscosity solution.

We also point out that in the recent work [1], in the case of the heat operator, in neighborhoods of free boundary points satisfying a certain flatness condition in some space direction, the free boundary was proved to be smooth (see Remark 8.5 ).

Concerning the assumptions on our quasilinear operator, we point out that at some stages we are able to improve our results in the particular case of an operator taking an isotropic form. In that case, we additionally assume that $A(p)=f(|p|)$ so we have $F(p)=\nabla A(p)=\left(f^{\prime}(|p|) /|p|\right) p$. In the free boundary problem $(P)$, we then get $\alpha(\nu, M)=\alpha(M)$-the free boundary condition is independent of $v$-and thus the free boundary problem becomes

$$
\begin{array}{ll}
\operatorname{div} F(\nabla u)-\partial_{t} u=0 & \text { in }\{u>0\}, \\
|\nabla u|=\alpha(M) & \text { on } \partial\{u>0\},
\end{array}
$$

where $\alpha=\alpha(M)$ is the unique positive number such that $-f(\alpha)+\alpha f^{\prime}(\alpha)=M$.

Our work also includes a section with some preliminary results (Section 3) and Appendix A, where we prove results on asymptotic developments at regular boundary points for nonnegative suband supersolutions to homogeneous quasilinear parabolic equations, used throughout the paper.

\section{Notation and assumptions}

We will assume that the function $F$ in equation $\left(P_{\varepsilon}\right)$ can be obtained as

$$
F(p)=\nabla A(p), \quad \forall p \in \mathbb{R}^{N},
$$

with

$$
A(0)=0 \quad \text { and } \quad A \in C^{3+\alpha}\left(\mathbb{R}^{N}, \mathbb{R}\right)
$$

for some $\alpha>0$. We will also assume that the operator

$$
\mathcal{L} u:=\operatorname{div} F(\nabla u)-\partial_{t} u
$$


is uniformly parabolic: there are constants $0<\vartheta_{\min } \leqslant \vartheta_{\max }$ such that

$$
\vartheta_{\min }|\xi|^{2} \leqslant \sum_{i, j=1}^{N} \frac{\partial F_{i}}{\partial p_{j}}(p) \xi_{i} \xi_{j} \leqslant \vartheta_{\max }|\xi|^{2} \quad \forall \xi, p \in \mathbb{R}^{N} .
$$

Observe that 2.4 implies

$$
(F(p)-F(q)) \cdot(p-q) \geqslant \vartheta_{\min }|p-q|^{2} \quad \forall p, q \in \mathbb{R}^{N} .
$$

We will also assume, without loss of generality, that $F(0)=0$, and thus the assumptions above imply that the function $A$ is nonnegative.

The functions $u^{\varepsilon}$ considered here will be weak solutions to $\left(P_{\varepsilon}\right)$ in a domain $\mathcal{D} \subseteq \mathbb{R}^{N+1}$. For the notion of weak solution we refer to [22, Chapter V]. We remark that, from our regularity assumptions on $F$ and $\beta$ it follows that, given a weak solution $u^{\varepsilon}$ of $\left(P_{\varepsilon}\right)$ in $\mathcal{D}$, we have $u^{\varepsilon} \in$ $H^{2+\alpha, 1+\alpha / 2}\left(\mathcal{D}^{\prime}\right)$ for every $\mathcal{D}^{\prime} \subset \subset \mathcal{D}$ (see [22]). Here we use the notation of [22] for Hölder spaces.

In addition, we will frequently consider solutions to the homogeneous equation

$$
\mathcal{L} u=\operatorname{div} F(\nabla u)-\partial_{t} u=0
$$

in some domain $\mathcal{D} \subseteq \mathbb{R}^{N+1}$. The regularity assumptions on $F$ above also imply that weak solutions to this equation are in $H^{3+\alpha,(3+\alpha) / 2}\left(\mathcal{D}^{\prime}\right)$ for any $\mathcal{D}^{\prime} \subset \subset \mathcal{D}$ (see, for instance, [19, Chapt. 3, Sect. 5]).

At some stages of the work we will restrict ourselves to an isotropic form of the operator $\mathcal{L}$. In that case we will additionally assume that

$$
A(p)=f(|p|), \quad A \text { as in 2.1 },
$$

for some function $f$ with $f(0)=f^{\prime}(0)=0$, and thus $F(p)=\left(f^{\prime}(|p|) /|p|\right) p$. If 2.6 is assumed, equation $\left(P_{\varepsilon}\right)$ takes the form

$$
\operatorname{div}\left(\frac{f^{\prime}\left(\left|\nabla u^{\varepsilon}\right|\right)}{\left|\nabla u^{\varepsilon}\right|} \nabla u^{\varepsilon}\right)-\partial_{t} u^{\varepsilon}=\beta_{\varepsilon}\left(u^{\varepsilon}\right) .
$$

We remark (see Lemma 3.1) that if 2.6 holds the function $F$ satisfies 2.4) if and only if

$$
\vartheta_{\min } \leqslant f^{\prime \prime}(s) \leqslant \vartheta_{\max } \quad \forall s>0,
$$

and 2.77 implies that $\vartheta_{\min } \leqslant f^{\prime}(s) / s \leqslant \vartheta_{\max }$ for $s>0$.

Throughout the paper $N$ will denote the spatial dimension and the following notation will be used:

For any $x_{0} \in \mathbb{R}^{N}, t_{0} \in \mathbb{R}$ and $\tau>0$,

$$
\begin{aligned}
& B_{\tau}\left(x_{0}\right):=\left\{x \in \mathbb{R}^{N}:\left|x-x_{0}\right|<\tau\right\}, \\
& B_{\tau}\left(x_{0}, t_{0}\right):=\left\{(x, t) \in \mathbb{R}^{N+1}:\left|x-x_{0}\right|^{2}+\left|t-t_{0}\right|^{2}<\tau^{2}\right\}, \\
& Q_{\tau}\left(x_{0}, t_{0}\right):=B_{\tau}\left(x_{0}\right) \times\left(t_{0}-\tau^{2}, t_{0}+\tau^{2}\right), \\
& Q_{\tau}^{-}\left(x_{0}, t_{0}\right):=B_{\tau}\left(x_{0}\right) \times\left(t_{0}-\tau^{2}, t_{0}\right],
\end{aligned}
$$


and for any set $K \subseteq \mathbb{R}^{N+1}$,

$$
\begin{aligned}
& \mathcal{N}_{\tau}(K):=\left\{(x, t):(x, t) \in Q_{\tau}\left(x_{0}, t_{0}\right) \text { for some }\left(x_{0}, t_{0}\right) \in K\right\}, \\
& \mathcal{N}_{\tau}^{-}(K):=\left\{(x, t):(x, t) \in Q_{\tau}^{-}\left(x_{0}, t_{0}\right) \text { for some }\left(x_{0}, t_{0}\right) \in K\right\} .
\end{aligned}
$$

When necessary, we denote points in $\mathbb{R}^{N}$ by $x=\left(x_{1}, x^{\prime}\right)$ with $x^{\prime} \in \mathbb{R}^{N-1}$. Also $\langle\cdot, \cdot\rangle$ will refer to the usual scalar product in $\mathbb{R}^{N}$. Given a function $v$ we write $v^{+}:=\max (v, 0)$. In addition the symbols $\nabla, \Delta$ and div will denote the corresponding operators in space variables and the symbol $\partial_{p}$ will denote the parabolic boundary.

We will say that a function $v$ is of class $\operatorname{Lip}_{\text {loc }}(1,1 / 2)$ in a domain $\mathcal{D} \subseteq \mathbb{R}^{N+1}$ if for every $\mathcal{D}^{\prime} \subset \subset \mathcal{D}$ there exists a constant $L=L\left(\mathcal{D}^{\prime}\right)$ such that

$$
|v(x, t)-v(y, \tau)| \leqslant L\left(|x-y|+|t-\tau|^{1 / 2}\right)
$$

for all $(x, t),(y, \tau)$ in $\mathcal{D}^{\prime}$. If the constant $L$ above does not depend on the set $\mathcal{D}^{\prime}$ we will say that $v \in \operatorname{Lip}(1,1 / 2)$ in $\mathcal{D}$.

We assume that the functions $\beta_{\varepsilon}$ are defined by scaling of a single function $\beta: \mathbb{R} \rightarrow \mathbb{R}$ satisfying

(1) $\beta$ is Lipschitz continuous,

(2) $\beta>0$ in $(0,1)$ and $\beta \equiv 0$ otherwise,

(3) $\int_{0}^{1} \beta(s) \mathrm{d} s=M$,

for some constant $M>0$. Finally, we define

$$
\beta_{\varepsilon}(s):=\frac{1}{\varepsilon} \beta\left(\frac{s}{\varepsilon}\right)
$$

and its primitive

$$
\mathcal{B}_{\varepsilon}(u):=\int_{0}^{u} \beta_{\varepsilon}(s) \mathrm{d} s .
$$

Observe that $\mathcal{B}_{\varepsilon}(u)=\int_{0}^{u} \beta_{\varepsilon}(s) \mathrm{d} s=\int_{0}^{u / \varepsilon} \beta(s) \mathrm{d} s \leqslant M$ and $\mathcal{B}_{\varepsilon}(\varepsilon)=M$.

\section{Preliminary results}

For the sake of completeness we collect in this section some preliminary results for the operator $\mathcal{L}$ defined in (2.3) that will be used throughout the paper.

We start with the following lemma, which deals with the isotropic case.

Lemma 3.1 Assume [2.6), which implies $F(p)=\left(f^{\prime}(|p|) /|p|\right) p$. Then the function $F$ satisfies (2.4) iff the function $f$ satisfies 2.7]. Moreover, 2.7] implies that $\vartheta_{\min } \leqslant f^{\prime}(s) / s \leqslant \vartheta_{\max }$ for $s>0$.

Proof. First observe that, since we have assumed that $f^{\prime}(0)=0$, 2.7) implies that $\vartheta_{\min } \leqslant$ $f^{\prime}(s) / s \leqslant \vartheta_{\max }$ for $s>0$.

Now observe that in the present case

$$
D F(p)=\left(f^{\prime \prime}(|p|)-\frac{f^{\prime}(|p|)}{|p|}\right) \frac{p \otimes p}{|p|^{2}}+\frac{f^{\prime}(|p|)}{|p|} \mathbb{I},
$$


and the constants $\vartheta_{\min }$ and $\vartheta_{\max }$ are bounds for the minimal and maximal eigenvalues of $D F(p)$. We notice that $p$ is the only nontrivial eigenvector of $p \otimes p$ and satisfies $(p \otimes p) p=|p|^{2} p$. Then the result follows from the fact that $D F(p) p=f^{\prime \prime}(|p|) p$ and that $D F(p) \xi=\left(f^{\prime}(|p|) /|p|\right) \xi$ for any $\xi$ orthogonal to $p$.

The rest of the results in the section deal with general operators. We get

LEMMA 3.2 (Comparison principle) Let $\Omega \subset \mathbb{R}^{N}$ be a bounded domain. Consider two functions $U$ and $w$, both in $H_{\text {loc }}^{1}(\Omega \times(0, T)) \cap C(\bar{\Omega} \times[0, T])$ such that $\mathcal{L} U \leqslant 0$ and $\mathcal{L} w \geqslant 0$ in $\Omega \times(0, T)$ and $U \geqslant w$ on $\partial_{p}(\Omega \times(0, T))$. Then $U \geqslant w$ in $\Omega \times(0, T)$.

Proof. Let $0<\tau<T$. Let $\psi \in H^{1}(\Omega \times(0, \tau)) \cap C(\bar{\Omega} \times[0, \tau])$ be a nonnegative function vanishing in a neighborhood of the parabolic boundary of $\Omega \times(0, \tau)$. We infer that

$$
\iint_{\Omega \times(0, \tau)}\left[(F(\nabla w)-F(\nabla U)) \cdot \nabla \psi+(w-U)_{t} \psi\right] \mathrm{d} x \mathrm{~d} t \leqslant 0 .
$$

We take $\delta>0$ and $\psi=(w-U-\delta)^{+}$, and use 2.5 to obtain

$$
\begin{aligned}
0 & \geqslant \iint_{\Omega \times(0, \tau)}\left[(F(\nabla w)-F(\nabla U)) \cdot \nabla(w-U-\delta)^{+}+(w-U-\delta)_{t}(w-U-\delta)^{+}\right] \mathrm{d} x \mathrm{~d} t \\
& \geqslant \iint_{\{w>U+\delta\} \cap\{t<\tau\}} \vartheta_{\min }|\nabla w-\nabla U|^{2} \mathrm{~d} x \mathrm{~d} t+\frac{1}{2} \iint_{\Omega \times(0, \tau)} \frac{\mathrm{d}}{\mathrm{d} t}\left[(w-U-\delta)^{+}\right]^{2} \mathrm{~d} x \mathrm{~d} t \\
& =\iint_{\{w>U+\delta\} \cap\{t<\tau\}} \vartheta_{\min }|\nabla w-\nabla U|^{2} \mathrm{~d} x \mathrm{~d} t+\frac{1}{2} \int_{\Omega}\left[(w(x, \tau)-U(x, \tau)-\delta)^{+}\right]^{2} \mathrm{~d} x,
\end{aligned}
$$

since $(w-U-\delta)^{+}=0$ on $\{t=0\}$. Hence both integrals are equal to zero. Since the choice of the time $\tau$ was arbitrary, the second integral being zero and the continuity of the functions then imply that $(w-U-\delta)^{+}=0$ in $\Omega \times[0, T]$. As the choice of $\delta>0$ was arbitrary, the result holds.

LEMMA 3.3 (Comparison principle in an unbounded domain) Let $U \in \operatorname{Lip}(1,1 / 2)$ be a solution to $\mathcal{L} U=0$ in the set $\mathcal{A}:=\left\{x_{1}>0\right\} \cap\{t>c\} \subset \mathbb{R}^{N+1}$, for some constant $c \in \mathbb{R}$, with $U=0$ on $\partial_{p} \mathcal{A}$. Then $U \leqslant 0$ in $\mathcal{A}$.

Proof. Let $L$ be the $\operatorname{Lip}(1,1 / 2)$ seminorm of $U$. For $R>0$, define $h_{R}(x, t):=(1 / R)\left(L|x|^{2}+\right.$ $\left.2 N L \vartheta_{\max }(t-c)\right)$ and observe that $\mathcal{L} h_{R} \leqslant 0$, as

$$
\begin{aligned}
\operatorname{div}\left(F\left(\nabla h_{R}\right)\right)-\frac{\partial h_{R}}{\partial t} & =\sum_{i=1}^{N} \frac{\partial F_{i}}{\partial p_{i}}\left(\frac{2 L x}{R}\right) \frac{2 L}{R}-\frac{2 N L \vartheta_{\max }}{R} \\
& \leqslant N \vartheta_{\max } \frac{2 L}{R}-\vartheta_{\max } \frac{2 N L}{R}=0 .
\end{aligned}
$$

Let $\mathcal{A}_{R}:=\mathcal{A} \cap\{|x| \leqslant R\}$ and notice that $U \leqslant h_{R}$ in $\partial_{p} \mathcal{A}_{R}$, since for $x$ such that $|x|=R$,

$$
U(x, t)=U\left(x_{1}, x^{\prime}, t\right)-U\left(0, x^{\prime}, t\right) \leqslant L x_{1} \leqslant L|x|=L \frac{|x|^{2}}{R} \leqslant h_{R}(x, t) .
$$

Then, by the comparison principle (see, for instance, Corollary 9.2 in [25]) it follows that $U \leqslant h_{R}$ in $\mathcal{A}_{R}$. Now, let $(y, s) \in \mathcal{A}$. Then for all $R \geqslant|y|$ we have $(y, s) \in \mathcal{A}_{R}$, and hence

$$
U(y, s) \leqslant h_{R}(y, s)=\frac{1}{R}\left(L|y|^{2}+2 N L \vartheta_{\max }(s-c)\right) .
$$

Letting $R \rightarrow \infty$, we get $U(y, s) \leqslant 0$. 
The comparison principle (Lemma 3.2) allows us to obtain

Lemma 3.4 Let $\mathcal{C}_{\tau}:=Q_{\tau}^{-}(0,0) \cap\left\{x_{1}>0\right\}$ and let $U \in H_{\text {loc }}^{1}\left(\mathcal{C}_{1}\right) \cap C\left(\overline{\mathcal{C}}_{1}\right)$ be such that $\mathcal{L} U \leqslant 0$ in $\mathcal{C}_{1}$, with $U>0$ in $\mathcal{C}_{1}$ and $U(0,0)=0$. Then

$$
U(x, t) \geqslant \alpha x_{1} \quad \text { in } \mathcal{C}_{\gamma}
$$

for some $\alpha>0$ and $0<\gamma<1$.

Proof. Let $\mathcal{A}=\Omega \times(-1,0)$, with $\Omega \subset B_{3 / 4}(0) \cap\left\{x_{1}>0\right\}$ a smooth domain, having $B_{1 / 2}(0) \cap$ $\left\{x_{1}=0\right\}$ as part of its boundary. Let $w \in H^{2+\alpha, 1+\alpha / 2}(\overline{\mathcal{A}})$ be such that $\mathcal{L} w=0$ in $\mathcal{A}$ and $w=\varphi$ on $\partial_{p} \mathcal{A}$, with $\varphi$ a smooth function such that $0<\varphi<U$ on $\partial_{p} \mathcal{A} \cap Q_{\tau}(\bar{x}, \bar{t})$ and $\varphi \equiv 0$ on $\partial_{p} \mathcal{A} \backslash Q_{\tau}(\bar{x}, \bar{t})$, for some $\bar{x} \in \partial \Omega \cap\left\{x_{1}>0\right\}, 0<\bar{t}<1$ and $\tau>0$ small enough. Then, in $\mathcal{C}_{\gamma}$,

$$
w\left(x_{1}, x^{\prime}, t\right)=w_{x_{1}}\left(0, x^{\prime}, t\right) x_{1}+o\left(x_{1}\right) \geqslant \alpha x_{1},
$$

for some $\alpha>0$ and $\gamma>0$ small, the last inequality being due to the application of the Hopf principle (Thm. 6, Chap. 3 in [26]).

Now, from Lemma 3.2 we deduce that $U \geqslant w$ in $\mathcal{A}$, and the result follows.

REMARK 3.1 Let $u$ be a solution to $\mathcal{L} u=0$ in $\mathcal{D} \subseteq \mathbb{R}^{N+1}$. Since, by our assumptions, $u \in$ $H^{3+\alpha,(3+\alpha) / 2}\left(\mathcal{D}^{\prime}\right)$ for any $\mathcal{D}^{\prime} \subset \subset \mathcal{D}$, we have $u_{t}=\operatorname{div} F(\nabla u)=\sum_{i, j} \frac{\partial F_{i}}{\partial p_{j}}(\nabla u) u_{x_{j} x_{i}}$ in $\mathcal{D}$, and thus, taking the derivative in direction $e_{k}$, we get

$$
\left(\frac{\partial u}{\partial x_{k}}\right)_{t}=\sum_{i, j} \frac{\partial F_{i}}{\partial p_{j}}(\nabla u)\left(\frac{\partial u}{\partial x_{k}}\right)_{x_{j} x_{i}}+\sum_{i, j, l} \frac{\partial^{2} F_{i}}{\partial p_{j} \partial p_{l}}(\nabla u)\left(\frac{\partial u}{\partial x_{k}}\right)_{x_{l}} u_{x_{j} x_{i}} \quad \text { in } \mathcal{D} .
$$

That is, $v=u_{x_{k}}$ satisfies the linear parabolic equation

$$
v_{t}=\sum_{i, j} a_{i j}(x, t) v_{x_{j} x_{i}}+\sum_{l} b_{l}(x, t) v_{x_{l}} \quad \text { in } \mathcal{D}
$$

with continuous coefficients $a_{i j}(x, t)=\frac{\partial F_{i}}{\partial p_{j}}(\nabla u)$ and $b_{l}(x, t)=\sum_{i, j} \frac{\partial^{2} F_{i}}{\partial p_{j} \partial p_{l}}(\nabla u) u_{x_{j} x_{i}}$.

If we now let $v=\frac{\partial u}{\partial v}$ for any vector $v \in \mathbb{S}^{N-1}$, we arrive at the same conclusion.

REMARK 3.2 Let $F$ be as in Section 2. Now, instead of the uniformly parabolic operator $\mathcal{L} u=$ $\operatorname{div} F(\nabla u)-\partial_{t} u$ defined in that section, let us consider the operator $\tilde{\mathcal{L}} u=\operatorname{div} \tilde{F}(\nabla u)-\partial_{t} u$ where $\tilde{F}(p)=-F(w-p)$, for some $w \in \mathbb{R}^{N}$ fixed.

Clearly, $\tilde{F}$ has the same smoothness as $F$. Moreover, if we let $q \in \mathbb{R}^{N}$, we notice that

$$
\frac{\partial \tilde{F}}{\partial q_{j}}(q)=\frac{\partial}{\partial q_{j}}(-F(w-q))=\frac{\partial F}{\partial p_{j}}(w-q),
$$

and therefore $\tilde{F}$ satisfies 2.4 and $\tilde{\mathcal{L}}$ is a uniformly parabolic operator as well.

We obtain the same conclusion if we consider instead the operator $\overline{\mathcal{L}} u=\operatorname{div} \bar{F}(\nabla u)-\partial_{t} u$ where $\bar{F}(p)=F(p-w)$.

\section{Uniform estimates and passage to the limit as $\varepsilon \rightarrow 0$}

In this section we consider a family $u^{\varepsilon}$ of nonnegative solutions to $\left(P_{\varepsilon}\right)$ in a domain $\mathcal{D} \subset \mathbb{R}^{N+1}$ which are uniformly bounded in $L^{\infty}$ norm in $\mathcal{D}$ and we obtain uniform estimates on the family $u^{\varepsilon}$ that allow the passage to the limit as $\varepsilon \rightarrow 0$. 
We also show that the limit function $u$ is a solution of the free boundary problem $(P)$ in a very weak sense. More precisely,

$$
\mathcal{L} u=\mu \quad \text { in } \mathcal{D},
$$

with $\mu$ a nonnegative Radon measure supported on the free boundary $\mathcal{D} \cap \partial\{u>0\}$.

We start by recalling Theorem 1 of [13].

PROPOSITION 4.1 Let $u^{\varepsilon}$ be a family of uniformly bounded nonnegative solutions of $\left(P_{\varepsilon}\right)$ in $B_{1}\left(x_{0}\right) \times(-1,0)$. Then this family is locally uniformly Lipschitz in space, i.e. there exists a constant $L$, depending only on the uniform bound of $\left\|u^{\varepsilon}\right\|_{L^{\infty}}$, such that

$$
\left|\nabla u^{\varepsilon}\right| \leqslant L \quad \text { in } B_{1 / 2}\left(x_{0}\right) \times(-1 / 2,0) .
$$

As a consequence we get

COROLLARY 4.1 Let $u^{\varepsilon}$ be a family of nonnegative solutions to $\left(P_{\varepsilon}\right)$ in a domain $\mathcal{D} \subset \mathbb{R}^{N+1}$ such that $\left\|u^{\varepsilon}\right\|_{L^{\infty}(\mathcal{D})} \leqslant \mathcal{A}$ for some $\mathcal{A}>0$. Let $K \subset \mathcal{D}$ be a compact set and let $\tau>0$ be such that $N_{\tau}^{-}(K) \subset \mathcal{D}$. There exists a constant $L=L(\tau, \mathcal{A})$ such that

$$
\left|\nabla u^{\varepsilon}\right| \leqslant L \quad \text { in } K .
$$

Proof. The proof is similar to the one of Corollary 2.1 in [14] and uses the fact that, for $\left(x_{0}, t_{0}\right) \in K$, the function $v_{\tau}^{\varepsilon}(x, t):=(1 / \tau) u^{\varepsilon}\left(x_{0}+\tau\left(x-x_{0}\right), t_{0}+\tau^{2} t\right)$ satisfies in the present case

$$
\operatorname{div} F\left(\nabla v_{\tau}^{\varepsilon}\right)-\partial_{t} v_{\tau}^{\varepsilon}=\beta_{\varepsilon / \tau}\left(v_{\tau}^{\varepsilon}\right) \quad \text { in } B_{1}\left(x_{0}\right) \times(-1,0) .
$$

The following lemma prepares the proof of Hölder $1 / 2$ continuity in time.

LEMMA 4.1 Let $u$ be a smooth nonnegative function in $\bar{B}_{1}(0) \times\left[0,1 /\left(4 N \vartheta_{\max }+\Lambda\right)\right]$ such that $|\mathcal{L} u| \leqslant \Lambda$ in $\{u>1\}$ for some $\Lambda>0$. Assume that $|\nabla u| \leqslant L$ for some $L>0$. Then there exists a constant $C=C(L)$ such that

$$
|u(0, T)-u(0,0)| \leqslant C \quad \text { for } 0 \leqslant T \leqslant \frac{1}{4 N \vartheta_{\max }+\Lambda} .
$$

Proof. The proof is an adaptation of the proof of Proposition 2.2 in [14]. Assume that $L>1$. We want to prove that if $Q_{t_{0}, t_{1}}:=B_{1}(0) \times\left(t_{0}, t_{1}\right) \subset\{u>1\}$ and $t_{1}-t_{0} \leqslant 1 /\left(4 N \vartheta_{\max }+\Lambda\right)$, then

$$
\left|u\left(0, t_{1}\right)-u\left(0, t_{0}\right)\right| \leqslant 2 L .
$$

In fact, let

$$
h^{ \pm}(x, t):=u\left(0, t_{0}\right) \pm L \pm 2 L|x|^{2} \pm\left(4 L N \vartheta_{\max }+\Lambda\right)\left(t-t_{0}\right) .
$$

Then

$$
h_{t}^{ \pm}-\operatorname{div} F\left(\nabla h^{ \pm}\right)= \pm\left(-4 L \operatorname{Tr}(D F( \pm 4 L x))+4 L N \vartheta_{\max }+\Lambda\right) .
$$

Observe that $\operatorname{Tr}(D F) \leqslant N \vartheta_{\max }$. Let

$$
t_{2}=\sup _{t_{0} \leqslant \bar{t} \leqslant t_{1}}\left\{\bar{t}:\left|u(0, t)-u\left(0, t_{0}\right)\right| \leqslant 2 L \forall t_{0} \leqslant t \leqslant \bar{t}\right\}
$$


and assume $t_{2}<t_{1}$. We compare $u$ with $h^{+}$and $h^{-}$in $Q_{t_{0}, t_{2}}$. By the Lipschitz continuity in space with Lipschitz constant $L$ we deduce that

$$
h^{-} \leqslant u \leqslant h^{+} \quad \text { in } \partial_{p} Q_{t_{0}, t_{2}} .
$$

On the other hand,

$$
h_{t}^{-}-\operatorname{div} F\left(\nabla h^{-}\right) \leqslant-\Lambda \leqslant u_{t}-\operatorname{div} F(\nabla u) \leqslant \Lambda \leqslant h_{t}^{+}-\operatorname{div} F\left(\nabla h^{+}\right) .
$$

Hence,

$$
h^{-} \leqslant u \leqslant h^{+} \quad \text { in } Q_{t_{0}, t_{2}} .
$$

In particular, since $t_{2}-t_{0}<t_{1}-t_{0} \leqslant 1 /\left(4 N \vartheta_{\max }+\Lambda\right)$,

$$
\begin{aligned}
\left|u\left(0, t_{2}\right)-u\left(0, t_{0}\right)\right| & \leqslant\left|h^{-}\left(0, t_{2}\right)-u\left(0, t_{0}\right)\right|=\left|h^{+}\left(0, t_{2}\right)-u\left(0, t_{0}\right)\right| \\
& <L+L \frac{4 N \vartheta_{\max }+\Lambda / L}{4 N \vartheta_{\max }+\Lambda}<2 L .
\end{aligned}
$$

The strict inequality contradicts the assumption $t_{2}<t_{1}$.

We now want to deduce the result for a cylinder $Q_{0, T}$ with $0<T \leqslant 1 /\left(4 N \vartheta_{\max }+\Lambda\right)$ which is not necessarily a subset of $\{u>1\}$. But this can be done exactly as in [14], and thus the proof is complete.

As a consequence we obtain the following theorem

THEOREM 4.1 Let $u^{\varepsilon}$ be a family of nonnegative solutions to $\left(P_{\varepsilon}\right)$ in a domain $\mathcal{D} \subset \mathbb{R}^{N+1}$ such that $\left\|u^{\varepsilon}\right\|_{L^{\infty}(\mathcal{D})} \leqslant \mathcal{A}$ for some $\mathcal{A}>0$. Let $K \subset \mathcal{D}$ be compact and let $\tau>0$ be such that $\mathcal{N}_{2 \tau}(K) \subset \mathcal{D}$. There exist constants $L=L(\tau, \mathcal{A})$ and $C=C(L, \tau, \mathcal{A})$ such that

$$
\left|\nabla u^{\varepsilon}(x, t)\right| \leqslant L \quad \text { and } \quad\left|u^{\varepsilon}(x, t+\Delta t)-u^{\varepsilon}(x, t)\right| \leqslant C|\Delta t|^{1 / 2} \quad \text { for }(x, t),(x, t+\Delta t) \in K .
$$

Proof. The proof follows the one of Theorem 2.1 in [14] and makes use of Corollary 4.1, Lemma 4.1, and the scaling invariance with respect to parabolic scaling of problem $\left(P_{\varepsilon}\right)$, observed in the proof of Corollary 4.1

We are now ready to pass to the limit. We obtain

LEMMA 4.2 Let $u^{\varepsilon}$ be a family of nonnegative solutions to $\left(P_{\varepsilon}\right)$ in a domain $\mathcal{D} \subset \mathbb{R}^{N+1}$ such that $\left\|u^{\varepsilon}\right\|_{L^{\infty}(\mathcal{D})} \leqslant \mathcal{A}$ for some $\mathcal{A}>0$. For every $\varepsilon_{n} \rightarrow 0$ there exists a subsequence $\varepsilon_{n^{\prime}} \rightarrow 0$ and $u \in \operatorname{Lip}_{\text {loc }}(1,1 / 2)(\mathcal{D})$ such that

(1) $u^{\varepsilon_{n^{\prime}}} \rightarrow u$ uniformly on compact subsets of $\mathcal{D}$,

(2) $\partial u^{\varepsilon_{n^{\prime}}} / \partial t \rightarrow \partial u / \partial t$ weakly in $L_{\text {loc }}^{2}(\mathcal{D})$,

(3) $\nabla u^{\varepsilon_{n^{\prime}}} \rightarrow \nabla u$ in $L_{\text {loc }}^{2}(\mathcal{D})$,

(4) $\mathcal{L} u \geqslant 0$ in $\mathcal{D}$,

(5) $\mathcal{L} u=0$ in $\mathcal{D} \backslash \partial\{u>0\}$,

(6) for every compact $K \subset \mathcal{D}$ there exists $C_{K}>0$ such that

$$
\left\|\frac{\partial u^{\varepsilon}}{\partial t}\right\|_{L^{2}(K)} \leqslant C_{K} \quad \text { for every } \varepsilon>0 .
$$


Proof. We modify the proof of Lemma 3.1 in [14]. Let $K \subset \mathcal{D}$ be compact and let $\tau>0$ be such that $\mathcal{N}_{3 \tau}(K) \subset \mathcal{D}$.

Point (1) is a consequence of the uniform Lipschitz continuity in space and Hölder continuity in time, by Theorem 4.1, and the Arzelà-Ascoli theorem.

For the proof of (2) we let $\left(x_{0}, t_{0}\right) \in K$ and multiply the equation $\left(P_{\varepsilon}\right)$ by $u_{t}^{\varepsilon} \psi^{2}$ where $\psi \in$ $C_{c}^{\infty}\left(B_{\tau}\left(x_{0}\right)\right), \psi \geqslant 0$ and $\psi \equiv 1$ on $B_{\tau / 2}\left(x_{0}\right)$. After integration by parts we obtain

$$
\begin{aligned}
& \iint_{Q_{\tau}\left(x_{0}, t_{0}\right)} \psi^{2}\left(u_{t}^{\varepsilon}\right)^{2} \mathrm{~d} x \mathrm{~d} t \\
& \quad=-\iint_{Q_{\tau}\left(x_{0}, t_{0}\right)}\left[2 \psi \nabla \psi \cdot F\left(\nabla u^{\varepsilon}\right) u_{t}^{\varepsilon}+\psi^{2} \frac{\partial}{\partial t}\left(A\left(\nabla u^{\varepsilon}\right)+\mathcal{B}_{\varepsilon}\left(u^{\varepsilon}\right)\right)\right] \mathrm{d} x \mathrm{~d} t \\
& \quad \leqslant 2\left\|\psi u_{t}^{\varepsilon}\right\|_{L^{2}\left(Q_{\tau}\left(x_{0}, t_{0}\right)\right)}\left\|\nabla \psi \cdot F\left(\nabla u^{\varepsilon}\right)\right\|_{L^{2}\left(Q_{\tau}\left(x_{0}, t_{0}\right)\right)}-\left[\int_{B_{\tau}\left(x_{0}\right)} \psi^{2}\left(A\left(\nabla u^{\varepsilon}\right)+\mathcal{B}_{\varepsilon}\left(u^{\varepsilon}\right)\right) \mathrm{d} x\right]_{t_{0}-\tau^{2}}^{t_{0}+\tau^{2}} \\
& \quad \leqslant C_{1}\left\|\psi u_{t}^{\varepsilon}\right\|_{L^{2}\left(Q_{\tau}\left(x_{0}, t_{0}\right)\right)}+C_{2},
\end{aligned}
$$

for some constants $C_{1}, C_{2}>0$, where we used the uniform bounds on $\nabla u^{\varepsilon}, F\left(\nabla u^{\varepsilon}\right)$ and $A\left(\nabla u^{\varepsilon}\right)$ and the definition 2.8. This implies the boundedness of $\left\|\psi u_{t}^{\varepsilon}\right\|_{L^{2}\left(Q_{\tau}\left(x_{0}, t_{0}\right)\right)}$ uniformly in $\varepsilon$. Since these bounds do not depend on the choice of $\left(x_{0}, t_{0}\right)$ in $K$, from the compactness of $K$ we conclude

$$
\left\|u_{t}^{\varepsilon}\right\|_{L^{2}(K)} \leqslant C
$$

where the constant $C$ does not depend on $\varepsilon$. The weak convergence of a subsequence $u_{t}^{\varepsilon_{n^{\prime}}}$ is an immediate consequence.

Let us prove (5). Since $u$ is continuous we may split the domain $\mathcal{D}$ as follows: $\mathcal{D}=\{u>0\}$ $\cup \partial\{u>0\} \cup\{u \equiv 0\}^{\circ}$, with $\{u>0\}$ open. It is obvious that $u$ satisfies $\mathcal{L} u=0$ in $\{u \equiv 0\}^{\circ}$. Since $u^{\varepsilon_{n^{\prime}}} \rightarrow u$ uniformly in $K$, for every $\left(x_{0}, t_{0}\right) \in\{u>0\}$ there is a neighborhood $\mathcal{U}\left(x_{0}, t_{0}\right) \subset K$ such that $u^{\varepsilon_{n^{\prime}}} \geqslant u\left(x_{0}, t_{0}\right) / 2>0$ in $\mathcal{U}\left(x_{0}, t_{0}\right)$. Therefore $\beta_{\varepsilon_{n^{\prime}}}\left(u^{\varepsilon_{n^{\prime}}}\right)=0$ on $\mathcal{U}\left(x_{0}, t_{0}\right)$ for $n^{\prime}$ large enough, which implies that $u^{\varepsilon_{n^{\prime}}}$ satisfies $\mathcal{L} u^{\varepsilon_{n^{\prime}}}=0$ on $\mathcal{U}\left(x_{0}, t_{0}\right)$, and the same holds for the limit $u$. Here we have used the fact that $\nabla u^{\varepsilon_{n^{\prime}}} \rightarrow \nabla u$ uniformly in $\mathcal{U}\left(x_{0}, t_{0}\right)$ (see, for instance, Theorem 12.1 in [25]).

Let us now prove (3). Since $\mathcal{L} u=0$ in $\{u>0\}$, if we let $\delta>0$ and multiply this equation by $(u-\delta)^{+} \psi(x)$, with $\psi$ as above, then integrate by parts in $Q_{\tau}\left(x_{0}, t_{0}\right)$ and let $\delta \rightarrow 0$, we get

$$
\begin{aligned}
\iint_{\{u>0\}} F(\nabla u) \cdot \nabla u \psi= & -\iint_{\{u>0\}} u F(\nabla u) \cdot \nabla \psi-\frac{1}{2} \int_{\{u>0\}} u^{2}\left(x, t_{0}+\tau^{2}\right) \psi(x) \\
& +\frac{1}{2} \int_{\{u>0\}} u^{2}\left(x, t_{0}-\tau^{2}\right) \psi(x) .
\end{aligned}
$$

On the other hand, since $\psi \geqslant 0$ and $\beta_{\varepsilon}\left(u^{\varepsilon}\right) u^{\varepsilon} \geqslant 0$,

$$
\begin{aligned}
\int_{t_{0}-\tau^{2}}^{t_{0}+\tau^{2}} \int_{B_{\tau}\left(x_{0}\right)} F\left(\nabla u^{\varepsilon}\right) \cdot \nabla u^{\varepsilon} \psi \leqslant & -\int_{t_{0}-\tau^{2}}^{t_{0}+\tau^{2}} \int_{B_{\tau}\left(x_{0}\right)} u^{\varepsilon} F\left(\nabla u^{\varepsilon}\right) \cdot \nabla \psi \\
& -\frac{1}{2} \int_{B_{\tau}\left(x_{0}\right)}\left(u^{\varepsilon}\right)^{2}\left(x, t_{0}+\tau^{2}\right) \psi(x) \\
& +\frac{1}{2} \int_{B_{\tau}\left(x_{0}\right)}\left(u^{\varepsilon}\right)^{2}\left(x, t_{0}-\tau^{2}\right) \psi(x),
\end{aligned}
$$


and therefore

$$
\limsup _{n^{\prime} \rightarrow \infty} \iint_{Q_{\tau}\left(x_{0}, t_{0}\right)} F\left(\nabla u^{\varepsilon_{n^{\prime}}}\right) \cdot \nabla u^{\varepsilon_{n^{\prime}}} \psi \leqslant \iint_{Q_{\tau}\left(x_{0}, t_{0}\right)} F(\nabla u) \cdot \nabla u \psi,
$$

which together with $(2.5)$ implies

$$
\lim _{n^{\prime} \rightarrow \infty} \iint_{Q_{\tau}\left(x_{0}, t_{0}\right)} F\left(\nabla u^{\varepsilon_{n^{\prime}}}\right) \cdot \nabla u^{\varepsilon_{n^{\prime}}} \psi=\iint_{Q_{\tau}\left(x_{0}, t_{0}\right)} F(\nabla u) \cdot \nabla u \psi .
$$

This and (2.5) finally yield

$$
\begin{aligned}
\vartheta_{\min } \lim _{n^{\prime} \rightarrow \infty} \iint_{Q_{\tau / 2}\left(x_{0}, t_{0}\right)}\left|\nabla u^{\varepsilon_{n^{\prime}}}-\nabla u\right|^{2} & \leqslant \lim _{n^{\prime} \rightarrow \infty} \vartheta_{\min } \iint_{Q_{\tau}\left(x_{0}, t_{0}\right)}\left|\nabla u^{\varepsilon_{n^{\prime}}}-\nabla u\right|^{2} \psi \\
& \leqslant \lim _{n^{\prime} \rightarrow \infty} \iint_{Q_{\tau}\left(x_{0}, t_{0}\right)}\left(F\left(\nabla u^{\varepsilon_{n^{\prime}}}\right)-F(\nabla u)\right) \cdot\left(\nabla u^{\varepsilon_{n^{\prime}}}-\nabla u\right) \psi \\
& =0,
\end{aligned}
$$

which implies (3).

Finally, we observe that $\mathcal{L} u^{\varepsilon_{n^{\prime}}} \geqslant 0$ and that, for a subsequence, $\nabla u^{\varepsilon_{n^{\prime}}} \rightarrow \nabla u$ pointwise in $K$. Therefore, $\mathcal{L} u \geqslant 0$, and the proof is now complete.

We next show that the limit function $u$ is a solution to a free boundary problem in a very weak sense.

Proposition 4.2 Let $u^{\varepsilon_{j}}$ be a family of nonnegative solutions to $\left(P_{\varepsilon_{j}}\right)$ in a domain $\mathcal{D} \subset \mathbb{R}^{N+1}$ such that $u^{\varepsilon_{j}} \rightarrow u$ uniformly on compact subsets of $\mathcal{D}$ and $\varepsilon_{j} \rightarrow 0$. There exists a nonnegative Radon measure $\mu$ supported on the free boundary $\mathcal{D} \cap \partial\{u>0\}$ such that $\beta_{\varepsilon_{j}}\left(u^{\varepsilon_{j}}\right) \rightarrow \mu$ as measures in $\mathcal{D}$, and therefore

$$
\mathcal{L} u=\mu \quad \text { in } \mathcal{D},
$$

that is, for every $\phi \in C_{c}^{\infty}(\mathcal{D})$,

$$
\iint_{\mathcal{D}}\left(u \phi_{t}-F(\nabla u) \cdot \nabla \phi\right) \mathrm{d} x \mathrm{~d} t=\iint_{\mathcal{D}} \phi \mathrm{d} \mu .
$$

Proof. We find uniform $L^{1}$ estimates for $\beta_{\varepsilon_{j}}\left(u^{\varepsilon_{j}}\right)$ and obtain (4.2) by proceeding as in [14, Proposition 3.1]. In the present case we use the uniform bound and the pointwise convergence (for a subsequence) of $\nabla u^{\varepsilon_{j}}$.

As a consequence of the convergence result (Lemma 4.2 , we obtain

LEMMA 4.3 Let $u^{\varepsilon_{j}}$ be a family of nonnegative solutions to $\left(P_{\varepsilon_{j}}\right)$ in a domain $\mathcal{D} \subset \mathbb{R}^{N+1}$ such that $u^{\varepsilon_{j}} \rightarrow u$ uniformly on compact subsets of $\mathcal{D}$ and $\varepsilon_{j} \rightarrow 0$. Let $\left(x_{0}, t_{0}\right) \in \mathcal{D} \cap \partial\{u>0\}$ and let $\left(x_{n}, t_{n}\right) \in \mathcal{D} \cap \partial\{u>0\}$ be such that $\left(x_{n}, t_{n}\right) \rightarrow\left(x_{0}, t_{0}\right)$ as $n \rightarrow \infty$. Let $\lambda_{n} \rightarrow 0$ and

$$
u_{\lambda_{n}}(x, t)=\frac{1}{\lambda_{n}} u\left(x_{n}+\lambda_{n} x, t_{n}+\lambda_{n}^{2} t\right), \quad\left(u^{\varepsilon_{j}}\right)_{\lambda_{n}}(x, t)=\frac{1}{\lambda_{n}} u^{\varepsilon_{j}}\left(x_{n}+\lambda_{n} x, t_{n}+\lambda_{n}^{2} t\right) .
$$

Assume that $u_{\lambda_{n}} \rightarrow U$ as $n \rightarrow \infty$ uniformly on compact subsets of $\mathbb{R}^{N+1}$. Then there exists $j(n) \rightarrow+\infty$ such that, for every $j_{n} \geqslant j(n)$ we have $\varepsilon_{j_{n}} / \lambda_{n} \rightarrow 0$ and 
(1) $\left(u^{\varepsilon_{j_{n}}}\right)_{\lambda_{n}} \rightarrow U$ uniformly on compact subsets of $\mathbb{R}^{N+1}$,

(2) $\nabla\left(u^{\varepsilon_{n}}\right)_{\lambda_{n}} \rightarrow \nabla U$ in $L_{\text {loc }}^{2}\left(\mathbb{R}^{N+1}\right)$,

(3) $\frac{\partial}{\partial t}\left(u^{\varepsilon_{j n}}\right)_{\lambda_{n}} \rightarrow \frac{\partial}{\partial t} U$ weakly in $L_{\text {loc }}^{2}\left(\mathbb{R}^{N+1}\right)$.

Also, we deduce that

(4) $\nabla u_{\lambda_{n}} \rightarrow \nabla U$ in $L_{\text {loc }}^{2}\left(\mathbb{R}^{N+1}\right)$,

(5) $\frac{\partial}{\partial t} u_{\lambda_{n}} \rightarrow \frac{\partial}{\partial t} U$ weakly in $L_{\text {loc }}^{2}\left(\mathbb{R}^{N+1}\right)$.

Proof. The lemma follows from the convergence result (Lemma 4.2), exactly as Lemma 3.2 in [14], since it does not rely on the specific structure of the equation.

In addition we get

LEMMA 4.4 Let $u^{\varepsilon_{j}}$ be a nonnegative solution to $\left(P_{\varepsilon_{j}}\right)$ in a domain $\mathcal{D}_{j} \subset \mathbb{R}^{N+1}$ with $\mathcal{D}_{j} \subset \mathcal{D}_{j+1}$ and $\bigcup_{j} \mathcal{D}_{j}=\mathbb{R}^{N+1}$ such that $u^{\varepsilon_{j}} \rightarrow U$ uniformly on compact subsets of $\mathbb{R}^{N+1}$ and $\varepsilon_{j} \rightarrow 0$. Assume that for some choice of positive numbers $d_{n}$ and points $\left(x_{n}, t_{n}\right) \in \partial\{U>0\}$, the sequence $U_{d_{n}}(x, t)=\left(1 / d_{n}\right) U\left(x_{n}+d_{n} x, t_{n}+d_{n}^{2} t\right)$ converges uniformly on compact subsets of $\mathbb{R}^{N+1}$ to a function $U_{0}$. Let $\left(u^{\varepsilon_{j}}\right)_{d_{n}}(x, t)=\left(1 / d_{n}\right) u^{\varepsilon_{j}}\left(x_{n}+d_{n} x, t_{n}+d_{n}^{2} t\right)$. Then there exists $j(n) \rightarrow+\infty$ such that, for every $j_{n} \geqslant j(n), \varepsilon_{j_{n}} / d_{n} \rightarrow 0$ and

(1) $\left(u^{\varepsilon_{j n}}\right)_{d_{n}} \rightarrow U_{0}$ uniformly on compact subsets of $\mathbb{R}^{N+1}$,

(2) $\nabla\left(u^{\varepsilon_{j_{n}}}\right)_{d_{n}} \rightarrow \nabla U_{0}$ in $L_{\mathrm{loc}}^{2}\left(\mathbb{R}^{N+1}\right)$,

(3) $\frac{\partial}{\partial t}\left(u^{\varepsilon_{j n}}\right)_{d_{n}} \rightarrow \frac{\partial}{\partial t} U_{0}$ weakly in $L_{\text {loc }}^{2}\left(\mathbb{R}^{N+1}\right)$.

Proof. We refer to the proof of Lemma 3.3 in [14].

We also obtain

LEMma 4.5 Let $u^{\varepsilon_{j}}$ be a family of nonnegative solutions to $\left(P_{\varepsilon_{j}}\right)$ in a domain $\mathcal{D} \subset \mathbb{R}^{N+1}$ with $\varepsilon_{j} \rightarrow 0$ and assume $\left\|u^{\varepsilon_{j}}\right\|_{L^{\infty}(\mathcal{D})} \leqslant \mathcal{A}$ for some $\mathcal{A}>0$. Then, for a subsequence, $\mathcal{B}_{\varepsilon_{j}}\left(u^{\varepsilon_{j}}\right) \rightarrow \chi$ in $L_{\text {loc }}^{1}(\mathcal{D})$, where $\chi(x, t) \in\{0, M\}$ a.e. in $\mathcal{D}$.

Proof. We will generalize the idea in the proof of Proposition 4.1 in [28] to our operator [2.3). In fact, let $\mathcal{U}:=B_{r}\left(x_{0}\right) \times\left(t_{1}, t_{2}\right) \subset \subset \mathcal{D}$ with $r>0$ so small that $\mathcal{U}^{\prime}:=B_{2 r}\left(x_{0}\right) \times\left(t_{1}, t_{2}\right) \subset \subset \mathcal{D}$. For simplicity we suppress the subscript $j$.

Let $\psi \in C_{c}^{\infty}(\mathcal{D})$ be such that $0 \leqslant \psi \leqslant 1$ and $\psi \equiv 1$ on $\mathcal{U}^{\prime}$. Then

$$
\begin{aligned}
\left\|\partial_{x_{i}} \mathcal{B}_{\varepsilon}\left(u^{\varepsilon}\right)\right\|_{L^{1}\left(\mathcal{U}^{\prime}\right)} & \leqslant \iint_{\mathcal{D}} \psi\left|\beta_{\varepsilon}\left(u^{\varepsilon}\right) \partial_{x_{i}} u^{\varepsilon}\right| \mathrm{d} x \mathrm{~d} t \leqslant C \iint_{\mathcal{D}} \beta_{\varepsilon}\left(u^{\varepsilon}\right) \psi \mathrm{d} x \mathrm{~d} t \\
& =C \iint_{\mathcal{D}}\left(\operatorname{div} F\left(\nabla u^{\varepsilon}\right)-u_{t}^{\varepsilon}\right) \psi \mathrm{d} x \mathrm{~d} t \\
& =C \iint_{\mathcal{D}}\left(-\nabla \psi \cdot F\left(\nabla u^{\varepsilon}\right)-u_{t}^{\varepsilon} \psi\right) \mathrm{d} x \mathrm{~d} t \leqslant C
\end{aligned}
$$

due to the uniform $L^{\infty}$ bound on $\nabla u^{\varepsilon}$ and the uniform $L^{2}$ bound on $u_{t}^{\varepsilon}$. Observe that an analogous computation for the time derivative fails since we do not control it in $L^{\infty}$.

Now, we define $e_{\varepsilon}:=A\left(\nabla u^{\varepsilon}\right)+\mathcal{B}_{\varepsilon}\left(u^{\varepsilon}\right)$ and we notice that

$$
\left\|e_{\varepsilon}\right\|_{L^{1}\left(\mathcal{U}^{\prime}\right)}=\iint_{\mathcal{U}^{\prime}}\left|A\left(\nabla u^{\varepsilon}\right)+\mathcal{B}_{\varepsilon}\left(u^{\varepsilon}\right)\right| \mathrm{d} x \mathrm{~d} t \leqslant(C+M) \mathcal{H}^{N+1}\left(\mathcal{U}^{\prime}\right)=C .
$$


Let $\phi_{\delta} \geqslant 0,0<\delta<r$, be a family of mollifiers such that $\operatorname{supp} \phi_{\delta} \subset B_{\delta}(0)$. For any $(x, t) \in \mathcal{U}$,

$$
\begin{aligned}
\partial_{t}\left(e_{\varepsilon} * \phi_{\delta}\right)(x, t)=\int_{\mathbb{R}^{N}}\left(F\left(\nabla u^{\varepsilon}\right) \cdot \nabla u_{t}^{\varepsilon}+\beta_{\varepsilon}\left(u^{\varepsilon}\right) u_{t}^{\varepsilon}\right)(y, t) \phi_{\delta}(x-y) \mathrm{d} y \\
\quad=\int_{\mathbb{R}^{N}}\left[F\left(\nabla u^{\varepsilon}\right) \cdot \nabla \phi_{\delta}(x-y) u_{t}^{\varepsilon}(y, t)+\left(-\operatorname{div} F\left(\nabla u^{\varepsilon}\right) u_{t}^{\varepsilon}+\beta_{\varepsilon}\left(u^{\varepsilon}\right) u_{t}^{\varepsilon}\right)(y, t) \phi_{\delta}(x-y)\right] \mathrm{d} y \\
\quad=\int_{\mathbb{R}^{N}} F\left(\nabla u^{\varepsilon}\right) \cdot \nabla \phi_{\delta}(x-y) u_{t}^{\varepsilon}(y, t) \mathrm{d} y-\int_{\mathbb{R}^{N}}\left(u_{t}^{\varepsilon}\right)^{2}(y, t) \phi_{\delta}(x-y) \mathrm{d} y \\
\quad \leqslant\left\|\nabla \phi_{\delta}\right\|_{L^{\infty}\left(\mathbb{R}^{N}\right)}\left\|F\left(\nabla u^{\varepsilon}\right)(t)\right\|_{L^{2}\left(B_{2 r}\left(x_{0}\right)\right)}\left\|u_{t}^{\varepsilon}(t)\right\|_{L^{2}\left(B_{2 r}\left(x_{0}\right)\right)} .
\end{aligned}
$$

Hence

$$
\iint_{\mathcal{U}}\left|\partial_{t}\left(e_{\varepsilon} * \phi_{\delta}\right)\right| \leqslant C_{1}^{\delta}
$$

Furthermore,

$$
\begin{aligned}
\int_{B_{r}\left(x_{0}\right)}\left|\nabla\left(e_{\varepsilon} * \phi_{\delta}\right)\right|(x, t) \mathrm{d} x & \leqslant \int_{B_{r}\left(x_{0}\right)} \int_{B_{\delta}(0)}\left|\nabla \phi_{\delta}(y)\right| e_{\varepsilon}(x-y, t) \mathrm{d} y \mathrm{~d} x \\
& \leqslant\left\|\nabla \phi_{\delta}\right\|_{L^{1}\left(\mathbb{R}^{N}\right)}\left\|e_{\varepsilon}(t)\right\|_{L^{1}\left(B_{2 r}\left(x_{0}\right)\right)}
\end{aligned}
$$

and thus

$$
\left\|\nabla\left(e_{\varepsilon} * \phi_{\delta}\right)\right\|_{L^{1}(\mathcal{U})} \leqslant\left\|\nabla \phi_{\delta}\right\|_{L^{1}\left(\mathbb{R}^{N}\right)}\left\|e_{\varepsilon}\right\|_{L^{1}\left(\mathcal{U}^{\prime}\right)} \leqslant C_{2}^{\delta} .
$$

Together with 4.4, 4.5) and by compact embedding, this implies that, for every $\delta$, the family $\left(e_{\varepsilon} * \phi_{\delta}\right)$ is precompact in $L^{1}(\mathcal{U})$.

On the other hand, we deduce from 4.3 that

$$
\left\|\mathcal{B}_{\varepsilon}\left(u^{\varepsilon}\right)-\mathcal{B}_{\varepsilon}\left(u^{\varepsilon}\right) * \phi_{\delta}\right\|_{L^{1}(\mathcal{U})} \leqslant C \delta .
$$

Since, for a subsequence, $A\left(\nabla u^{\varepsilon_{j}}\right)$ converges in $L^{1}\left(\mathcal{U}^{\prime}\right)$, we also find that

$$
\sup _{j}\left\|A\left(\nabla u^{\varepsilon_{j}}\right)-A\left(\nabla u^{\varepsilon_{j}}\right) * \phi_{\delta}\right\|_{L^{1}(\mathcal{U})} \rightarrow 0 \quad \text { as } \delta \rightarrow 0 .
$$

Then, using a diagonal sequence argument we conclude that, for a subsequence, $\mathcal{B}_{\varepsilon_{j}}\left(u^{\varepsilon_{j}}\right) \rightarrow \chi$ in $L_{\text {loc }}^{1}(\mathcal{D})$. Finally, the limit function satisfies $\chi(x, t) \in\{0, M\}$ a.e. in $\mathcal{D}$, as in the proof of Lemma $4.1 \mathrm{in}[28]$.

REMARK 4.1 We point out that existence of families $u^{\varepsilon}$ of uniformly bounded nonnegative solutions to $\left(P_{\varepsilon}\right)$ in particular domains $\mathcal{D}$-and such that the results in this section and the subsequent ones apply—can be easily obtained. We refer, for instance, to [25, Chap. XII, Sect. 6] and to [22, Chap. V, Sect. 6].

\section{Basic limits}

In this section we analyze some particular limits of problems $\left(P_{\varepsilon}\right)$ that will be crucial to understanding the behavior of general limits. In order to deal with these particular limits we need to introduce a function $\Phi_{v}$ (Definition 5.1) and discuss its properties (Lemma 5.1). 
We study, in particular, the case in which the limit function is $u=\alpha\left\langle x-x_{0}, v\right\rangle^{+}$for some unit vector $v \in \mathbb{R}^{N}$ and $\alpha>0$ (Theorem 5.1). We characterize its slope as $\alpha=\alpha(v, M)$ (see (5.4)). This characterization will give us the free boundary condition of the limit problem $(P)$ in the general case (we refer to the subsequent sections for the precise assumptions and results).

We also present some examples of different operators $\mathcal{L}$ and we exhibit the resulting free boundary condition.

Definition 5.1 Let $v \in \mathbb{R}^{N}$ be a unit vector. We define $\Phi_{v}: \mathbb{R}_{\geqslant 0} \rightarrow \mathbb{R}_{\geqslant 0}$ by

$$
\Phi_{\nu}(\alpha):=-A(\alpha \nu)+\alpha \nu \cdot F(\alpha \nu) .
$$

Lemma 5.1 The function $\Phi_{v}$ given in Definition 5.1 has the following properties:

(1) $\Phi_{v}(0)=0$.

(2) It is a strictly increasing function in $\alpha$ and therefore invertible.

(3) It satisfies

$$
\frac{\alpha^{2}}{2} \vartheta_{\min } \leqslant \Phi_{\nu}(\alpha) \leqslant \frac{\alpha^{2}}{2} \vartheta_{\max } \quad \text { for every } \alpha \geqslant 0 .
$$

(4) In case (2.6) holds, the function $\Phi_{v}$ does not depend on the direction $v$ and

$$
\Phi_{\nu}(\alpha)=-f(\alpha)+\alpha f^{\prime}(\alpha) .
$$

Proof. The properties are either straightforward to observe or a consequence of the fact that $\Phi_{v}^{\prime}(\alpha)=\alpha \nu \cdot D F(\alpha \nu) v$ and thus, by [2.4), $\alpha \vartheta_{\min } \leqslant \Phi_{\nu}^{\prime}(\alpha) \leqslant \alpha \vartheta_{\max }$.

THEOREM 5.1 Let $u^{\varepsilon_{j}}$ be nonnegative solutions to $\left(P_{\varepsilon_{j}}\right)$ in a domain $\mathcal{D} \subset \mathbb{R}^{N+1}$ and let $\left(x_{0}, t_{0}\right) \in \mathcal{D}$. Suppose that $u^{\varepsilon_{j}}$ converge to $u=\alpha\left\langle x-x_{0}, v\right\rangle^{+}$uniformly on compact subsets of $\mathcal{D}$, for some unit vector $v \in \mathbb{R}^{N}$ and $\alpha>0$, and $\varepsilon_{j} \rightarrow 0$. Then $\alpha=\alpha(\nu, M)$, with

$$
\alpha(v, M):=\Phi_{v}^{-1}(M), \quad \Phi_{v} \text { as in } 5.1 \text {. }
$$

Moreover,

$$
\sqrt{\frac{2 M}{\vartheta_{\max }}} \leqslant \alpha(v, M) \leqslant \sqrt{\frac{2 M}{\vartheta_{\min }}} .
$$

Proof. We will assume that $\left(x_{0}, t_{0}\right)=(0,0)$. Let us multiply $\left(P_{\varepsilon}\right)$ by $\psi u_{x_{i}}^{\varepsilon} \nu^{i}=\psi \nabla u^{\varepsilon} \cdot v$ where $\psi \in C_{c}^{\infty}(\mathcal{D})$. Integration by parts yields

$$
\iint_{\mathcal{D}}\left(F\left(\nabla u^{\varepsilon}\right) \cdot\left[\psi \nabla u_{x_{i}}^{\varepsilon} \nu^{i}+\nabla \psi \nabla u^{\varepsilon} \cdot v\right]+u_{t}^{\varepsilon} \psi \nabla u^{\varepsilon} \cdot v\right) \mathrm{d} x \mathrm{~d} t=-\iint_{\mathcal{D}} \nabla \mathcal{B}_{\varepsilon}\left(u^{\varepsilon}\right) \cdot v \psi \mathrm{d} x \mathrm{~d} t,
$$

where we have used (2.8). Hence

$$
\begin{aligned}
& \iint_{\mathcal{D}} u_{t}^{\varepsilon} \psi \nabla u^{\varepsilon} \cdot v \mathrm{~d} x \mathrm{~d} t \\
& \quad=\iint_{\mathcal{D}}\left(-\frac{\partial}{\partial x_{i}}\left(A\left(\nabla u^{\varepsilon}\right)\right) \psi v^{i}-F\left(\nabla u^{\varepsilon}\right) \cdot \nabla \psi \nabla u^{\varepsilon} \cdot v\right) \mathrm{d} x \mathrm{~d} t+\iint_{\mathcal{D}} \mathcal{B}_{\varepsilon}\left(u^{\varepsilon}\right) \nabla \psi \cdot v \mathrm{~d} x \mathrm{~d} t \\
& \quad=\iint_{\mathcal{D}}\left[A\left(\nabla u^{\varepsilon}\right) \nabla \psi \cdot v-F\left(\nabla u^{\varepsilon}\right) \cdot \nabla \psi \nabla u^{\varepsilon} \cdot v+\mathcal{B}_{\varepsilon}\left(u^{\varepsilon}\right) \nabla \psi \cdot v\right] \mathrm{d} x \mathrm{~d} t \\
& \quad=\iint_{\mathcal{D}}\left[\left(A\left(\nabla u^{\varepsilon}\right)+\mathcal{B}_{\varepsilon}\left(u^{\varepsilon}\right)\right) v-\nabla u^{\varepsilon} \cdot v F\left(\nabla u^{\varepsilon}\right)\right] \cdot \nabla \psi \mathrm{d} x \mathrm{~d} t
\end{aligned}
$$


Lemma 4.5 shows that (for a subsequence) $\mathcal{B}_{\varepsilon_{j}}\left(u^{\varepsilon_{j}}\right) \rightarrow M \chi_{\{\langle x, v\rangle>0\}}+\bar{M}(x, t) \chi_{\{\langle x, v\rangle<0\}}$ in $L_{\text {loc }}^{1}(\mathcal{D})$ with $M(x, t)=0$ or $\bar{M}(x, t)=M$ almost everywhere. Here we have also used the fact that if $(y, \tau) \in \mathcal{D} \cap\{u>0\}$, then $u^{\varepsilon_{j}}(x, t) \geqslant u(y, \tau) / 2>0$ for $(x, t)$ in a neighborhood of $(y, \tau)$ if $j$ is large enough and therefore, $\mathcal{B}_{\varepsilon_{j}}\left(u^{\varepsilon_{j}}\right)(x, t)=M$ in this neighborhood for $j$ large. On the other hand, since $\nabla \mathcal{B}_{\varepsilon_{j}}\left(u^{\varepsilon_{j}}\right) \rightarrow 0$ in $L_{\text {loc }}^{1}\left(\mathcal{D} \cap\{u \equiv 0\}^{\circ}\right)$ (recall that $\beta_{\varepsilon_{j}}\left(u^{\varepsilon_{j}}\right) \rightarrow 0$ as measures in $\mathcal{D} \cap\{u \equiv 0\}^{\circ}$ by Proposition 4.2 we deduce that $\bar{M}(x, t)=\bar{M}(t)$. Now, since $\nabla u^{\varepsilon_{j}} \rightarrow \alpha \nu \chi_{\{\langle x, v\rangle>0\}}$ in $L_{\text {loc }}^{2}(\mathcal{D})$ and $u_{t}^{\varepsilon_{j}} \rightarrow 0$ weakly in $L_{\text {loc }}^{2}(\mathcal{D})$, as $\varepsilon_{j} \rightarrow 0$, making use of 2.2. we obtain in the limit

$$
\begin{aligned}
0 & =\iint_{\langle x, \nu\rangle>0}[(A(\alpha \nu)+M) \nu-\alpha \nu \cdot v F(\alpha \nu)] \cdot \nabla \psi \mathrm{d} x \mathrm{~d} t+\iint_{\langle x, \nu\rangle<0} \bar{M}(t) \nu \cdot \nabla \psi \mathrm{d} x \mathrm{~d} t \\
& =\iint_{\langle x, \nu\rangle=0}[(-A(\alpha \nu)-M+\bar{M}(t)) \nu+\alpha F(\alpha \nu)] \cdot \nu \psi \mathrm{d} \mathcal{H}^{n-1}(x) \mathrm{d} t .
\end{aligned}
$$

Hence, using Definition 5.1 , we get

$$
\Phi_{v}(\alpha)=M-\bar{M}(t) .
$$

Since the left hand side in 5.7) does not depend on $t$, we see that $\bar{M}(t)=\bar{M}$, with $\bar{M}=M$ or $\bar{M}=0$. If $\bar{M}=M$, then the monotonicity of $\Phi_{\nu}$ implies that $\alpha=0$, a contradiction. Hence

$$
\Phi_{v}(\alpha)=M
$$

and the monotonicity of $\Phi_{v}$ now implies that for every $v$ there is a unique solution to $(5.8)$ given by [5.4). Finally, the estimates (5.2) give (5.5).

REMARK 5.1 Notice that defining $\Phi(p):=-A(p)+p \cdot F(p)$, we can also write 5.8) as $\Phi(\alpha \nu)=M$.

Next we present some examples of different operators $\mathcal{L}$ and we exhibit the resulting free boundary condition.

EXAMPLE 5.1 Let $A(p)=|p|^{2} / 2$. Then $F(p)=p$ and we obtain $\alpha=\sqrt{2 M}$.

EXAMPLE 5.2 Let $A(p)=\frac{1}{2} p \cdot B p$ for some symmetric, positive definite matrix $B \in \mathbb{R}^{N \times N}$. Then $F(p)=B p$ and we obtain $\alpha=\sqrt{2 M /(v \cdot B v)}$. In particular, if $B$ is a positive multiple of the identity, $B=c \operatorname{Id}_{N \times N}$ with $c>0$, we obtain $\alpha=\sqrt{2 M / c}$ as the free boundary condition.

EXAMPLE 5.3 If 2.6 holds (and thus $A(p)=f(|p|)$ and $F(p)=\left(f^{\prime}(|p|) /|p|\right) p$, with $f$ satisfying (2.7)), then the slope $\alpha$ at the free boundary does not depend on the direction $v$. With abuse of notation we write in this case

$$
\alpha(M)=\alpha(v, M), \quad \alpha(v, M) \text { as in } 5.4),
$$

and, since (5.3) holds, we observe that $\alpha(M)$ is the solution of

$$
-f(\alpha)+\alpha f^{\prime}(\alpha)=M .
$$

EXAMPLE 5.4 We finally observe that, when $A(p)=|p|^{q} / q$ with $q>1, q \neq 2$ and thus $F(p)=$ $|p|^{q-2} p$, the condition $\alpha=\alpha(v, M)$ gives $\alpha=\left(\frac{q M}{q-1}\right)^{1 / q}$. However, this choice of $A$ and $F$ does not in general satisfy (2.4) nor our smoothness assumptions. 
In the isotropic case we obtain, in addition,

THEOREM 5.2 Let $u^{\varepsilon_{j}}$ be nonnegative solutions to $\left(P_{\varepsilon_{j}}\right)$ in a domain $\mathcal{D} \subset \mathbb{R}^{N+1}$ and assume 2.6 holds. Let $\left(x_{0}, t_{0}\right) \in \mathcal{D}$ and suppose that $u^{\varepsilon_{j}}$ converge to $u=\alpha\left(x-x_{0}\right)_{1}^{+}+\bar{\alpha}\left(x-x_{0}\right)_{1}^{-}$with $\alpha>0$, $\bar{\alpha}>0$ uniformly on compact subsets of $\mathcal{D}$ and $\varepsilon_{j} \rightarrow 0$. Then

$$
\alpha=\bar{\alpha} \leqslant \alpha(M)
$$

where $\alpha(M)$ is given by 5.9 . .

Proof. We follow the lines of Proposition 5.3 in [14]. Set $Q_{r}=Q_{r}(0,0)$ and assume $\left(x_{0}, t_{0}\right)=$ $(0,0)$ and $Q_{2} \subset \subset \mathcal{D}$ (take $\bar{u}(x, t):=(1 / \lambda) u\left(x_{0}+\lambda x, t_{0}+\lambda^{2} t\right)$ with $\lambda$ small). Reasoning as in Theorem 5.1, we obtain

$$
\mathcal{B}_{\varepsilon_{j}}\left(u^{\varepsilon_{j}}\right) \rightarrow M \quad \text { in } L_{\mathrm{loc}}^{1}(\mathcal{D}) .
$$

Going back to 5.6, letting $v=e_{1}$ there, and making use of the fact that $\nabla u^{\varepsilon_{j}} \rightarrow \alpha \chi_{\left\{x_{1}>0\right\}} e_{1}-$ $\bar{\alpha} \chi_{\left\{x_{1}<0\right\}} e_{1}$ in $L_{\mathrm{loc}}^{2}(\mathcal{D})$ and $u_{t}^{\varepsilon_{j}} \rightarrow 0$ weakly in $L_{\mathrm{loc}}^{2}(\mathcal{D})$, in the limit we obtain

$$
\begin{aligned}
0= & \iint_{\left\{x_{1}<0\right\}}\left[\left(A\left(-\bar{\alpha} e_{1}\right)+M\right) e_{1}+\bar{\alpha} e_{1} \cdot e_{1} F\left(-\bar{\alpha} e_{1}\right)\right] \cdot \nabla \psi \mathrm{d} x \mathrm{~d} t \\
& +\iint_{\left\{x_{1}>0\right\}}\left[\left(A\left(\alpha e_{1}\right)+M\right) e_{1}-\alpha e_{1} \cdot e_{1} F\left(\alpha e_{1}\right)\right] \cdot \nabla \psi \mathrm{d} x \mathrm{~d} t \\
= & \iint_{\left\{x_{1}=0\right\}}\left[A\left(-\bar{\alpha} e_{1}\right) e_{1}+\bar{\alpha} F\left(-\bar{\alpha} e_{1}\right)-A\left(\alpha e_{1}\right) e_{1}+\alpha F\left(\alpha e_{1}\right)\right] \cdot e_{1} \psi \mathrm{d} \mathcal{H}^{n-1}(x) \mathrm{d} t .
\end{aligned}
$$

Using Definition 5.1 we obtain $\Phi_{e_{1}}(\alpha)=\Phi_{-e_{1}}(\bar{\alpha})$, which gives

$$
\Phi_{e_{1}}(\alpha)=\Phi_{e_{1}}(\bar{\alpha}),
$$

and thus $\alpha=\bar{\alpha}$. We remark again that the function $\Phi_{\nu}$ does not depend on the direction $v$ in this case and is given by (5.3). To show the inequality in 5.11) we assume $\Phi_{e_{1}}(\alpha)>M$. Let

$$
b^{\varepsilon_{j}}=\sup _{Q_{2}}\left|u^{\varepsilon_{j}}-u\right| .
$$

Now let $v^{\varepsilon_{j}}$ be the solutions to $\left(P_{\varepsilon_{j}}\right)$ in $Q_{2}$ such that

$$
v^{\varepsilon_{j}}=\left(u-b^{\varepsilon_{j}}\right)^{+} \quad \text { on } \partial_{p} Q_{2} .
$$

Since $v^{\varepsilon_{j}}$ is a family of uniformly bounded nonnegative solutions to $\left(P_{\varepsilon_{j}}\right)$ in $Q_{2}$, it follows from Lemma 4.2 that there exists $v \in \operatorname{Lip}_{\text {loc }}(1,1 / 2)$ in $Q_{2}$ such that, for a subsequence, $v^{\varepsilon_{j}} \rightarrow v$ uniformly on compact subsets of $Q_{2}$. We will show that $v=u$. From the fact that $u^{\varepsilon_{j}} \geqslant v^{\varepsilon_{j}}$ on $\partial_{p} Q_{2}$, we deduce that $u^{\varepsilon_{j}} \geqslant v^{\varepsilon_{j}}$ in $Q_{2}$ and therefore $u \geqslant v$.

In order to see that $u \leqslant v$, let $g(s)=F\left(s e_{1}\right) \cdot e_{1}$ and choose $w \in C^{2}(\mathbb{R})$ such that

$$
\left(g\left(w^{\prime}(s)\right)\right)^{\prime}=\beta(w(s)), \quad s \in \mathbb{R}, \quad w(0)=1, w^{\prime}(0)=\alpha
$$

(such a $w$ exists by our assumptions). Multiply by $w^{\prime}(s)$ and observe that

$$
\left(\Phi_{e_{1}}\left(w^{\prime}(s)\right)\right)^{\prime}=\left(g\left(w^{\prime}(s)\right)\right)^{\prime} w^{\prime}(s)=\mathcal{B}(w(s))^{\prime},
$$


where $\mathcal{B}(\tau)=\int_{0}^{\tau} \beta(s) \mathrm{d} s$. For every $s \in \mathbb{R}$ by integration we obtain

$$
\Phi_{e_{1}}\left(w^{\prime}(s)\right)-\mathcal{B}(w(s))=\Phi_{e_{1}}(\alpha)-M=\Phi_{e_{1}}(\gamma), \quad \gamma>0
$$

(here we have used the fact that $\Phi_{e_{1}}(\alpha)>M$ ). Therefore $w^{\prime}(s)>0$ in $\mathbb{R}$ and moreover, since $\Phi_{e_{1}}$ is monotone in $\mathbb{R} \geqslant 0$, we conclude that

$$
0<\gamma \leqslant w^{\prime}(s) \leqslant \alpha \quad \forall s \in \mathbb{R} .
$$

It follows that there is $\bar{s}<0$ such that

$$
w(s)= \begin{cases}1+\alpha s, & s \geqslant 0 \\ \gamma(s-\bar{s}), & s \leqslant \bar{s}\end{cases}
$$

Now let

$$
w^{\varepsilon_{j}}\left(x_{1}\right)=\varepsilon_{j} w\left(\frac{1}{\varepsilon_{j}}\left(x_{1}-\frac{b^{\varepsilon_{j}}}{\gamma}\right)+\bar{s}\right) .
$$

Using the fact that $w^{\varepsilon_{j}}(0)=-b^{\varepsilon_{j}}$ and the bounds on $w^{\prime}$, we deduce that

$$
w^{\varepsilon_{j}} \leqslant u-b^{\varepsilon_{j}} \quad \text { in } \mathbb{R} .
$$

Hence $w^{\varepsilon_{j}}$ is a family of solutions to $\left(P_{\varepsilon_{j}}\right)$ in $\mathbb{R}^{N+1}$, satisfying $w^{\varepsilon_{j}} \leqslant v^{\varepsilon_{j}}$ on $\partial_{p} Q_{2}$. Therefore $w^{\varepsilon_{j}} \leqslant v^{\varepsilon_{j}}$ in $Q_{2}$. Since $w^{\varepsilon_{j}} \rightarrow u$ uniformly on compact subsets of $\left\{x_{1}>0\right\}$, we deduce that $u \leqslant v$ in $Q_{2} \cap\left\{x_{1}>0\right\}$. Finally, by the symmetry $v^{\varepsilon_{j}}\left(x_{1}, x^{\prime}, t\right)=v^{\varepsilon_{j}}\left(-x_{1}, x^{\prime}, t\right)$, we conclude that $u \leqslant v$ in $Q_{2}$.

Next, let

$$
\mathcal{R}:=\left\{(x, t): 0<x_{1}<1,\left|x^{\prime}\right|<1,|t|<1\right\} .
$$

Multiply $\left(P_{\varepsilon_{j}}\right)$ by $v_{x_{1}}^{\varepsilon_{j}}$. Integration yields

$$
\begin{aligned}
\iint_{\mathcal{R}} \frac{\partial}{\partial x_{1}} A\left(\nabla v^{\varepsilon_{j}}\right) & =\iint_{\mathcal{R}} F\left(\nabla v^{\varepsilon_{j}}\right) \cdot \nabla v_{x_{1}}^{\varepsilon_{j}} \\
& =\iint_{\mathcal{R}}\left(-v_{t}^{\varepsilon_{j}} v_{x_{1}}^{\varepsilon_{j}}-\frac{\partial}{\partial x_{1}} \mathcal{B}_{\varepsilon_{j}}\left(v^{\varepsilon_{j}}\right)\right)+\int_{\partial \mathcal{R}} v_{x_{1}}^{\varepsilon_{j}} F\left(\nabla v^{\varepsilon_{j}}\right) \cdot n_{x} \mathrm{~d} S
\end{aligned}
$$

and

$$
\begin{aligned}
& \int_{\partial \mathcal{R} \cap\left\{x_{1}=1\right\}}\left(A\left(\nabla v^{\varepsilon_{j}}\right)-v_{x_{1}}^{\varepsilon_{j}} F\left(\nabla v^{\varepsilon_{j}}\right) \cdot e_{1}+\mathcal{B}_{\varepsilon_{j}}\left(v^{\varepsilon_{j}}\right)\right) \mathrm{d} x^{\prime} \mathrm{d} t \\
& \quad=\int_{\partial \mathcal{R} \cap\left\{x_{1}=0\right\}}\left(A\left(\nabla v^{\varepsilon_{j}}\right)+\mathcal{B}_{\varepsilon_{j}}\left(v^{\varepsilon_{j}}\right)\right) \mathrm{d} x^{\prime} \mathrm{d} t+\iint_{\mathcal{R}}-v_{t}^{\varepsilon_{j}} v_{x_{1}}^{\varepsilon_{j}}+\int_{\partial \mathcal{R} \cap\left\{\left|x^{\prime}\right|=1\right\}} v_{x_{1}}^{\varepsilon_{j}} F\left(\nabla v^{\varepsilon_{j}}\right) \cdot n \mathrm{~d} S \\
& \quad \geqslant \iint_{\mathcal{R}}-v_{t}^{\varepsilon_{j}} v_{x_{1}}^{\varepsilon_{j}}+\int_{\partial \mathcal{R} \cap\left\{\left|x^{\prime}\right|=1\right\}} v_{x_{1}}^{\varepsilon_{j}} F\left(\nabla v^{\varepsilon_{j}}\right) \cdot n \mathrm{~d} S .
\end{aligned}
$$

The inequality holds since the functions $\mathcal{B}_{\varepsilon_{j}}$ and $A$ are nonnegative. Observe that we have used the equality $v_{x_{1}}^{\varepsilon_{j}}=0$ on $\left\{x_{1}=0\right\}$ due to the symmetry of $v^{\varepsilon_{j}}$. The first integral on the right hand side converges to zero since $v_{t}^{\varepsilon_{j}} \rightarrow u_{t}=0$ weakly in $L^{2}\left(Q_{3 / 2}\right)$ and $\nabla v^{\varepsilon_{j}} \rightarrow \nabla u$ in $L^{2}\left(Q_{3 / 2}\right)$. 
Additionally we infer that $v_{x_{1}}^{\varepsilon_{j}} F\left(\nabla v^{\varepsilon_{j}}\right) \rightarrow \alpha F\left(\alpha e_{1}\right)$ pointwise on $Q_{3 / 2} \cap\left\{x_{1}>0\right\}$ and $n\left(x_{1}, x^{\prime}, t\right)=$ $-n\left(x_{1},-x^{\prime}, t\right)$ on $\left\{\left|x^{\prime}\right|=1\right\}$, hence the remaining integral converges to zero. Furthermore, observe that $\mathcal{B}_{\varepsilon_{j}}\left(v^{\varepsilon_{j}}\right) \equiv M$ on $Q_{3 / 2} \cap\left\{x_{1}>1 / 2\right\}$, since $v^{\varepsilon_{j}}>\varepsilon_{j}$ if $\varepsilon_{j}$ is small enough. In the limit we therefore obtain

$$
M \geqslant-A\left(\alpha e_{1}\right)+\alpha F\left(\alpha e_{1}\right) \cdot e_{1}=\Phi_{e_{1}}(\alpha),
$$

which is a contradiction to our assumption.

\section{Upper bounds for the gradient of limit functions}

In this section we prove upper bounds for the gradient of limit functions, related to the free boundary condition. In the particular case of an isotropic operator this upper bound says that any limit function is, in a sense that we may call pseudo-classical, a supersolution to the free boundary problem $(P)$, without imposing any additional hypothesis (Theorem 6.1).

We first consider the isotropic case and we obtain

THEOREM 6.1 Let $u^{\varepsilon_{j}}$ be nonnegative solutions to $\left(P_{\varepsilon_{j}}\right)$ in a domain $\mathcal{D} \subset \mathbb{R}^{N+1}$ and assume 2.6 holds. Suppose $u^{\varepsilon_{j}} \rightarrow u$ uniformly on compact subsets of $\mathcal{D}$ and $\varepsilon_{j} \rightarrow 0$, and let $\left(x_{0}, t_{0}\right) \in$ $\mathcal{D} \cap \partial\{u>0\}$. Then

$$
\limsup _{(x, t) \rightarrow\left(x_{0}, t_{0}\right)}|\nabla u(x, t)| \leqslant \alpha(M),
$$

where $\alpha(M)$ is given by 5.9 .

Proof. We will follow the lines of Theorem 6.1 in [14]. Let

$$
\alpha:=\limsup _{(x, t) \rightarrow\left(x_{0}, t_{0}\right)}|\nabla u(x, t)| .
$$

Since $u \in \operatorname{Lip}_{\text {loc }}(1,1 / 2)$ in $\mathcal{D}$, we know that $\alpha<\infty$. If $\alpha=0$ there is nothing to prove. So let us assume that $\alpha>0$ and let $\left(x_{n}, t_{n}\right) \rightarrow\left(x_{0}, t_{0}\right)$ be such that $u\left(x_{n}, t_{n}\right)>0$ and $\left|\nabla u\left(x_{n}, t_{n}\right)\right| \rightarrow \alpha$. Let $\left(z_{n}, s_{n}\right) \in \mathcal{D} \cap \partial\{u>0\}$ be such that

$$
d_{n}:=\max \left\{\left|x_{n}-z_{n}\right|,\left|t_{n}-s_{n}\right|^{1 / 2}\right\}=\inf _{(z, s) \in \partial\{u>0\}}\left\{\max \left\{\left|x_{n}-z\right|,\left|t_{n}-s\right|^{1 / 2}\right\}\right\} .
$$

Let us consider the sequence

$$
u_{d_{n}}(x, t):=\frac{1}{d_{n}} u\left(z_{n}+d_{n} x, s_{n}+d_{n}^{2} t\right)
$$

Since $u \in \operatorname{Lip}_{\text {loc }}(1,1 / 2)$ in $\mathcal{D}$ and $d_{n} \rightarrow 0$, given a compact set $K \subset \mathbb{R}^{N+1}$ the functions $u_{d_{n}}$ are uniformly bounded with respect to the $\operatorname{Lip}(1,1 / 2)$ seminorm on $K$, if $n$ is large enough. On the other hand, $u_{d_{n}}(0,0)=0$ for every $n$. This implies that the family $u_{d_{n}}$ is uniformly bounded on compact subsets of $\mathbb{R}^{N+1}$. Hence for a subsequence (that we will still call $u_{d_{n}}$ ), $u_{d_{n}} \rightarrow u_{0}$ uniformly on compact subsets of $\mathbb{R}^{N+1}$, where $u_{0} \in \operatorname{Lip}(1,1 / 2)$ in $\mathbb{R}^{N+1}$.

Let $\bar{x}_{n}:=\left(x_{n}-z_{n}\right) / d_{n}$ and $\bar{t}_{n}:=\left(t_{n}-s_{n}\right) / d_{n}^{2}$. Then $\left(\bar{x}_{n}, \bar{t}_{n}\right) \in \partial Q_{1}(0,0)$ so that (for a subsequence), $\bar{x}_{n} \rightarrow \bar{x}$ and $\bar{t}_{n} \rightarrow \bar{t}$ with $(\bar{x}, \bar{t}) \in \partial Q_{1}(0,0)$. On the other hand, since $u_{d_{n}}>0$ in $Q_{1}\left(\bar{x}_{n}, \bar{t}_{n}\right)$, we have $\mathcal{L} u_{d_{n}}=0$ there. Let us show that $\nabla u_{d_{n}} \rightarrow \nabla u_{0}$ uniformly on compact subsets of $Q_{1}(\bar{x}, \bar{t})$. 
In fact, if $K \subset \subset Q_{1}(\bar{x}, \bar{t})$ then, for some $\tau>0$ and for $n$ large, $\mathcal{N}_{\tau}(K) \subset Q_{1}\left(\bar{x}_{n}, \bar{t}_{n}\right)$ and thus $\mathcal{L} u_{d_{n}}=0$ in $\mathcal{N}_{\tau}(K)$. It then follows that $\nabla u_{d_{n}} \rightarrow \nabla u_{0}$ uniformly in $K$ (see, for instance, Theorem 12.1 in [25]).

Therefore we deduce that, in $Q_{1}(\bar{x}, \bar{t}), u_{0}(x, t) \geqslant 0$ and $\mathcal{L} u_{0}=0$.

Now consider the sequence $v_{n}:=\nabla u_{d_{n}}\left(\bar{x}_{n}, \bar{t}_{n}\right) /\left|\nabla u_{d_{n}}\left(\bar{x}_{n}, \bar{t}_{n}\right)\right|=\nabla u\left(x_{n}, t_{n}\right) /\left|\nabla u\left(x_{n}, t_{n}\right)\right|$. We may assume (by taking a subsequence) that $v_{n} \rightarrow v$. From the uniform convergence of the gradients we deduce that

$$
\left|\nabla u\left(x_{n}, t_{n}\right)\right|=\frac{\partial u_{d_{n}}}{\partial v_{n}}\left(\bar{x}_{n}, \bar{t}_{n}\right) \rightarrow \frac{\partial u_{0}}{\partial v}(\bar{x}, \bar{t}),
$$

and therefore

$$
\frac{\partial u_{0}}{\partial v}(\bar{x}, \bar{t})=\alpha .
$$

On the other hand, it is easy to see that $\left|\nabla u_{0}\right| \leqslant \alpha$ in $\mathbb{R}^{N+1}$. In fact, let $R>0$ and $\delta>0$ be fixed. There exists $\lambda_{0}$ such that

$$
|\nabla u(x, t)| \leqslant \alpha+\delta \quad \text { for }(x, t) \in Q_{\lambda R}\left(x_{0}, t_{0}\right)
$$

if $\lambda \leqslant \lambda_{0}$. Since $Q_{d_{n} R}\left(z_{n}, s_{n}\right) \subset Q_{3 \lambda_{n} R}\left(x_{0}, t_{0}\right)$ if $\lambda_{n}=\max \left\{\left|x_{n}-x_{0}\right|,\left|t_{n}-t_{0}\right|^{1 / 2}\right\}\left(\geqslant d_{n}\right)$, and $R>1$ and since $\lambda_{n} \rightarrow 0$ as $n \rightarrow \infty$, we deduce that

$$
\left|\nabla u_{d_{n}}(x, t)\right| \leqslant \alpha+\delta \quad \text { for }(x, t) \in Q_{R}(0,0)
$$

if $n$ is large enough. Thus $\nabla u_{d_{n}} \rightarrow \nabla u_{0}$ in the weak* topology on $L^{\infty}\left(Q_{R}(0,0)\right)$ and therefore $\left|\nabla u_{0}\right| \leqslant \alpha+\delta$ in $Q_{R}(0,0)$. Since $\delta$ and $R$ were arbitrary we deduce that

$$
\left|\nabla u_{0}\right| \leqslant \alpha \quad \text { in } \mathbb{R}^{N+1} .
$$

Let $V:=\partial u_{0} / \partial v$ and observe that $V \leqslant \alpha$ in $\left\{u_{0}>0\right\}$ and $V(\bar{x}, \bar{t})=\alpha$. Since $\alpha>0$ we must have $u_{0}(\bar{x}, \bar{t})>0$, because otherwise $u_{0} \equiv 0$ in $Q_{1}^{-}(\bar{x}, \bar{t})$, due to the strong maximum principle, as $\mathcal{L} u_{0}=0$ in this set (see, for instance, [26, Chap. 3, Sect. 3]). Thus $u_{0}>0$ in $Q_{\rho}(\bar{x}, \bar{t})$ for some $\rho>0$. This fact, together with Remark 3.1 allows us to apply the same strong maximum principle to $V$ in $Q_{\rho}(\bar{x}, \bar{t})$ and conclude that $V \equiv \alpha$ in $Q_{\rho}^{-}(\bar{x}, \bar{t})$. Moreover, if we denote by $\mathcal{R}$ the set of points in $\left\{u_{0}>0\right\} \cap\{t<\bar{t}\}$ which can be connected to $(\bar{x}, \bar{t})$ by a continuous curve in $\left\{u_{0}>0\right\}$ along which the $t$-coordinate is nondecreasing, we see that $V \equiv \alpha$ in $\mathcal{R}$.

Now using the fact that $\mathcal{L} u_{0}=0$ in $\mathcal{R}$ and reasoning as in [14], we deduce that $\mathcal{R}=\left\{(x-\tilde{x})_{1}>0\right.$, $t<\bar{t}\}$ for some $\tilde{x} \in \mathbb{R}^{N}$, and

$$
u_{0}(x, t)=\alpha(x-\tilde{x})_{1} \quad \text { in }\left\{(x-\tilde{x})_{1}>0, t<\bar{t}\right\}
$$

(we have assumed that $v=e_{1}$ ). Let

$$
\left(u^{\varepsilon_{j}}\right)_{d_{n}}(x, t)=\frac{1}{d_{n}} u^{\varepsilon_{j}}\left(z_{n}+d_{n} x, s_{n}+d_{n}^{2} t\right) .
$$

By Lemma 4.3 there exists a sequence $j_{n} \rightarrow \infty$ such that $\left(u^{\varepsilon_{j n}}\right)_{d_{n}} \rightarrow u_{0}$ uniformly on compact subsets of $\mathbb{R}^{N+1}$ and $\varepsilon_{j_{n}} / d_{n} \rightarrow 0$. It is easy to see that $\left(u^{\varepsilon_{j_{n}}}\right)_{d_{n}}$ is a solution to $\left(P_{\varepsilon_{j_{n}} / d_{n}}\right)$ in $Q_{1}(\tilde{x}, \bar{t})$, if $n$ is large. 
Let us now apply Corollary A.1 of Appendix $\mathrm{A}$ to $u_{0}$ in $\left\{(x-\tilde{x})_{1}<0, t<\bar{t}\right\}$. We get, for some $\bar{\alpha} \geqslant 0$,

$$
u_{0}(x, t)=\bar{\alpha}(x-\tilde{x})_{1}^{-}+o\left(|x-\tilde{x}|+|t-\bar{t}|^{1 / 2}\right) \quad \text { in }\left\{(x-\tilde{x})_{1}<0, t<\bar{t}\right\} .
$$

Let us consider for $\lambda>0$ the function $\left(u_{0}\right)_{\lambda}(x, t)=(1 / \lambda) u_{0}\left(\lambda x+\tilde{x}, \lambda^{2} t+\bar{t}\right)$. Since $u_{0} \in$ $\operatorname{Lip}(1,1 / 2)$ and $u_{0}(\tilde{x}, \bar{t})=0$, there exists a sequence $\lambda_{k} \rightarrow 0$ such that $\left(u_{0}\right)_{\lambda_{k}}$ converges uniformly on compact subsets of $\mathbb{R}^{N+1}$ to a function $u_{00} \in \operatorname{Lip}(1,1 / 2)$ in $\mathbb{R}^{N+1}$. Thus,

$$
u_{00}(x, t)=\alpha x_{1}^{+}+\bar{\alpha} x_{1}^{-} \quad \text { in }\{t \leqslant 0\} .
$$

If we set $\varepsilon_{n}^{0}=\varepsilon_{j_{n}} / d_{n}$ and $u^{\varepsilon_{n}^{0}}=\left(u^{\varepsilon_{j n}}\right)_{d_{n}}$ then $u^{\varepsilon_{n}^{0}}$ are solutions to $P_{\varepsilon_{n}^{0}}$ in $Q_{1}(\tilde{x}, \bar{t})$ such that

$$
\begin{array}{ll}
u^{\varepsilon_{n}^{0}} \rightarrow u_{0} & \text { as } n \rightarrow \infty \text { uniformly on compact subsets of } Q_{1}(\tilde{x}, \bar{t}), \\
\left(u_{0}\right)_{\lambda_{k}} \rightarrow u_{00} & \text { as } k \rightarrow \infty \text { uniformly on compact subsets of } \mathbb{R}^{N+1},
\end{array}
$$

and $\varepsilon_{n}^{0} \rightarrow 0$ and $\lambda_{k} \rightarrow 0$. Therefore we can apply Lemma 4.3 again and find a sequence $\varepsilon_{n}^{00} \rightarrow 0$ and solutions $u^{\varepsilon_{n}^{00}}$ to $\left(P_{\varepsilon_{n}^{00}}\right)$ in $Q_{1}(0,0)$ such that

$$
u^{\varepsilon_{n}^{00}} \rightarrow u_{00}=\alpha x_{1}^{+}+\bar{\alpha} x_{1}^{-} \quad \text { uniformly on compact subsets of } Q_{1}^{-}(0,0) .
$$

If $\bar{\alpha}=0$ we apply Theorem 5.1 and if $\bar{\alpha}>0$ we apply Theorem 5.2. In any case, we deduce that $\alpha \leqslant \alpha(M)$. Thus the theorem is proved.

Also in the isotropic case we get

THEOREM 6.2 Let $u^{\varepsilon_{j}}$ be nonnegative solutions to $\left(P_{\varepsilon_{j}}\right)$ in a domain $\mathcal{D}_{j} \subset \mathbb{R}^{N+1}$ such that $\mathcal{D}_{j} \subset$ $\mathcal{D}_{j+1}$ and $\bigcup_{j} \mathcal{D}_{j}=\mathbb{R}^{N+1}$, and assume (2.6) holds. Suppose that $u^{\varepsilon_{j}} \rightarrow U$ uniformly on compact subsets of $\mathbb{R}^{N+1}$ and $\varepsilon_{j} \rightarrow 0$. Assume in addition that $U \in \operatorname{Lip}(1,1 / 2)$ in $\mathbb{R}^{N+1}$ and $\partial\{U>0\} \neq \emptyset$. Then

$$
|\nabla U| \leqslant \alpha(M) \quad \text { in } \mathbb{R}^{N+1},
$$

where $\alpha(M)$ is given by 5.9 .

Proof. We modify the proof of Theorem 6.2 in [14] in the same way as we generalised the proof of Theorem 6.1 in [14] in the case of the previous result, Theorem 6.1.

For a general operator we obtain

THEOREM 6.3 Let $u^{\varepsilon_{j}}$ be nonnegative solutions to $\left(P_{\varepsilon_{j}}\right)$ in a domain $\mathcal{D} \subset \mathbb{R}^{N+1}$ such that $\mathcal{D}_{j} \subset$ $\mathcal{D}_{j+1}$ and $\bigcup_{j} \mathcal{D}_{j}=\mathbb{R}^{N+1}$. Suppose that $u^{\varepsilon_{j}} \rightarrow U$ uniformly on compact subsets of $\mathbb{R}^{N+1}$ and $\varepsilon_{j} \rightarrow 0$. Assume in addition that $U \in \operatorname{Lip}(1,1 / 2)$ in $\mathbb{R}^{N+1}, U>0$ in $\left\{x_{1}>0\right\}$ and $U \equiv 0$ in $\left\{x_{1}<0\right\}$. Then

$$
|\nabla U| \leqslant \alpha\left(e_{1}, M\right) \quad \text { in } \mathbb{R}^{N+1},
$$

where $\alpha\left(e_{1}, M\right)$ is given by (5.4). 
Proof. We follow the steps of Theorem 6.2 in [14]. Let $\alpha:=\sup |\nabla U|$. By assumption $\alpha<\infty$. If $\alpha=0$ there is nothing to prove, so assume $\alpha>0$. Then there exist $\left(x_{n}, t_{n}\right)$ with $U\left(x_{n}, t_{n}\right)>0$ such that $\left|\nabla U\left(x_{n}, t_{n}\right)\right| \rightarrow \alpha$ as $n \rightarrow \infty$. Let $\left(z_{n}, s_{n}\right) \in \partial\{U>0\}$ be such that

$$
d_{n}:=\max \left\{\left|x_{n}-z_{n}\right|,\left|t_{n}-s_{n}\right|^{1 / 2}\right\}=\inf _{(z, s) \in \partial\{U>0\}}\left\{\max \left\{\left|x_{n}-z\right|,\left|t_{n}-s\right|^{1 / 2}\right\}\right\}
$$

and observe that since $\partial\{U>0\}=\left\{x_{1}=0\right\}$ we may write $\left(x_{n}, t_{n}\right)=\left(x_{n, 1}, x_{n}^{\prime}, t_{n}\right),\left(z_{n}, s_{n}\right)=$ $\left(0, x_{n}^{\prime}, t_{n}\right)$ and $d_{n}=x_{n, 1}$. We define

$$
U_{d_{n}}(x, t):=\frac{1}{d_{n}} U\left(z_{n}+d_{n} x, s_{n}+d_{n}^{2} t\right)
$$

and we observe that $U_{d_{n}}$ is uniformly bounded in $\operatorname{Lip}(1,1 / 2)$ seminorm and $U_{d_{n}}(0,0)=0$, so, for a subsequence, $U_{d_{n}} \rightarrow U_{0}$ uniformly on compact subsets of $\mathbb{R}^{N+1}$, with $U_{0} \in \operatorname{Lip}(1,1 / 2)$ in $\mathbb{R}^{N+1}$. Notice that $U_{0} \equiv 0$ in $\left\{x_{1}<0\right\}$, since this holds for $U$ and for $U_{d_{n}}$. Moreover, $U_{d_{n}}>0$ in $Q_{1}\left(e_{1}, 0\right)$ and hence $\mathcal{L} U_{d_{n}}=0$ there, so $\mathcal{L} U_{0}=0$ in $Q_{1}\left(e_{1}, 0\right)$.

Furthermore, for a subsequence,

$$
\frac{\nabla U\left(x_{n}, t_{n}\right)}{\left|\nabla U\left(x_{n}, t_{n}\right)\right|}=\frac{\nabla U_{d_{n}}\left(e_{1}, 0\right)}{\left|\nabla U_{d_{n}}\left(e_{1}, 0\right)\right|} \rightarrow v
$$

for some unit vector $v$, and since

$$
\nabla U_{d_{n}}\left(e_{1}, 0\right) \cdot \frac{\nabla U_{d_{n}}\left(e_{1}, 0\right)}{\left|\nabla U_{d_{n}}\left(e_{1}, 0\right)\right|}=\left|\nabla U_{d_{n}}\left(e_{1}, 0\right)\right| \rightarrow \alpha,
$$

we infer $\frac{\partial U_{0}}{\partial v}\left(e_{1}, 0\right)=\alpha$. Also, since $\left|\nabla U_{d_{n}}(x, t)\right|=\left|\nabla U\left(z_{n}+d_{n} x, s_{n}+d_{n}^{2} t\right)\right| \leqslant \alpha$, we obtain $\frac{\partial U_{0}}{\partial v}(x, t) \leqslant\left|\nabla U_{0}(x, t)\right| \leqslant \alpha$. We let $V:=\partial U_{0} / \partial v$ and we deduce, with similar arguments to those in the proof of Theorem 6.1 that $V \equiv \alpha$ in $\mathcal{R} \supset Q_{1}^{-}\left(e_{1}, 0\right)$, with $\mathcal{R}$ being the set of points of $\left\{U_{0}>0\right\} \cap\{t<0\}$ which can be connected to $\left(e_{1}, 0\right)$ by a continuous curve in $\left\{U_{0}>0\right\}$ along which the $t$-coordinate is nondecreasing. Then $U_{0}(x, t)=\alpha\langle x, v\rangle+C$ in $\mathcal{R}$ for a constant $C \in \mathbb{R}$, and $U_{0}(0,0)=0$ implies that $C=0$. Finally, the fact that $U_{0} \equiv 0$ in $\left\{x_{1}<0\right\}$ implies that $v=e_{1}$ and hence

$$
U_{0}(x, t)=\alpha x_{1}^{+} \quad \text { in }\{t \leqslant 0\},
$$

where $\alpha=\alpha\left(e_{1}, M\right)$ by Theorem 5.1 .

\section{Asymptotic development at free boundary points}

In this section we prove that, under suitable assumptions, the free boundary condition is satisfied in a pointwise sense. This happens either at free boundary points where there is an inward normal in the parabolic measure-theoretic sense (Theorem 7.1), or at free boundary points where the free boundary is locally a differentiable surface (Theorem 7.2), provided a nondegeneracy condition at the point holds.

DEFINITION 7.1 We say that $v \in \mathbb{S}^{N-1}$ is the inward unit spatial normal to the free boundary $\partial\{u>0\}$ at a point $\left(x_{0}, t_{0}\right) \in \partial\{u>0\}$ for times $t \leqslant t_{0}$, in the parabolic measure-theoretic sense, if

$$
\lim _{r \rightarrow 0^{+}} \frac{1}{r^{N+2}} \iint_{Q_{r}^{-}\left(x_{0}, t_{0}\right)}\left|\chi_{\{u>0\}}-\chi_{\left\{(x, t):\left\langle x-x_{0}, \nu\right\rangle>0\right\}}\right| \mathrm{d} x \mathrm{~d} t=0 .
$$

In this case the point $\left(x_{0}, t_{0}\right) \in \partial\{u>0\}$ will be called regular. 
Definition 7.2 We say that $u$ is nondegenerate at $\left(x_{0}, t_{0}\right) \in \mathcal{D} \cap \partial\{u>0\}$ if there exists a constant $C>0$ such that, for every $r>0$ small,

$$
\sup _{\partial_{p} Q_{r}^{-}\left(x_{0}, t_{0}\right)} u \geqslant C r .
$$

In the isotropic case we get

THEOREM 7.1 Let $u^{\varepsilon_{j}}$ be nonnegative solutions to $\left(P_{\varepsilon_{j}}\right)$ in a domain $\mathcal{D} \subset \mathbb{R}^{N+1}$ and assume 2.6 holds. Suppose that $u^{\varepsilon_{j}} \rightarrow u$ uniformly on compact subsets of $\mathcal{D}$ and $\varepsilon_{j} \rightarrow 0$. Let $\left(x_{0}, t_{0}\right) \in$ $\mathcal{D} \cap \partial\{u>0\}$ be a regular point and assume that $u$ is nondegenerate at $\left(x_{0}, t_{0}\right)$. Then, for $t \leqslant t_{0}$,

$$
u(x, t)=\alpha(M)\left\langle x-x_{0}, v\right\rangle^{+}+o\left(\left|x-x_{0}\right|+\left|t-t_{0}\right|^{1 / 2}\right),
$$

where $v$ is the inward unit spatial normal to the free boundary $\partial\{u>0\}$ at $\left(x_{0}, t_{0}\right)$ for times $t \leqslant t_{0}$, in the parabolic measure-theoretic sense, and $\alpha(M)$ is given by (5.9).

Proof. We follow the lines of Theorem 7.1 in [24]. We assume that $\left(x_{0}, t_{0}\right)=(0,0)$ and $v=e_{1}$. Let

$$
u_{\lambda}(x, t):=\frac{1}{\lambda} u\left(\lambda x, \lambda^{2} t\right)
$$

and let $r>0$ be such that $Q_{r}(0,0) \subset \subset \mathcal{D}$. We have $u_{\lambda} \in \operatorname{Lip}(1,1 / 2)$ in $Q_{r / \lambda}(0,0)$ uniformly in $\lambda$, and $u_{\lambda}(0,0)=0$. Therefore, for every $\lambda_{n} \rightarrow 0$, there exists a subsequence, which we still call $\lambda_{n}$, and a function $U \in \operatorname{Lip}(1,1 / 2)$ in $\mathbb{R}^{N+1}$ such that $u_{\lambda_{n}} \rightarrow U$ uniformly on compact subsets of $\mathbb{R}^{N+1}$. $\lambda \rightarrow 0$,

Our aim is to prove that $U=\alpha(M) x_{1}^{+}$for $t \leqslant 0$. By 7.1 , it follows that for every $k>0$, as

$$
\left|\left\{u_{\lambda}>0\right\} \cap\left\{x_{1}<0\right\} \cap Q_{k}^{-}(0,0)\right| \rightarrow 0, \quad\left|\left\{u_{\lambda} \equiv 0\right\} \cap\left\{x_{1}>0\right\} \cap Q_{k}^{-}(0,0)\right| \rightarrow 0 .
$$

This implies that $U \equiv 0$ in $\left\{x_{1}<0\right\} \cap\{t \leqslant 0\}$. On the other hand, $U$ is nonnegative, $\mathcal{L} U \geqslant 0$ and $\mathcal{L} U=0$ in $\{U>0\}$, with $\{U>0\} \cap\{t<0\} \subset\left\{x_{1}>0\right\}$. Then, by Corollary A.1 there exists $\alpha \geqslant 0$ such that

$$
U(x, t)=\alpha x_{1}^{+}+o\left(|x|+|t|^{1 / 2}\right) \quad \text { in }\left\{x_{1}>0\right\} \cap\{t<0\} .
$$

The nondegeneracy assumption on $u$ at $\left(x_{0}, t_{0}\right)$ implies that necessarily $\alpha>0$. Let us now show that $\alpha=\alpha(M)$. By Lemma 4.3 there exists a subsequence $\varepsilon_{j_{n}}$ such that $\delta_{n}:=\varepsilon_{j_{n}} / \lambda_{n} \rightarrow 0$ and $u^{\delta_{n}}(x, t):=\left(1 / \lambda_{n}\right) u^{\varepsilon_{j_{n}}}\left(\lambda_{n} x, \lambda_{n}^{2} t\right) \rightarrow U(x, t)$ uniformly on compact subsets of $\mathbb{R}^{N+1}$ and $u^{\delta_{n}}$ is a solution to $\left(P_{\delta_{n}}\right)$.

Now let $U_{\lambda}(x, t)=(1 / \lambda) U\left(\lambda x, \lambda^{2} t\right)$. Then for a sequence $\lambda_{k} \rightarrow 0, U_{\lambda_{k}} \rightarrow \alpha x_{1}^{+}$in $\{t \leqslant 0\}$, uniformly on compact subsets. As before, there exists a subsequence $\delta_{n_{k}}$ such that $\bar{\delta}_{k}:=\delta_{n_{k}} / \lambda_{k} \rightarrow 0$ and $u^{\bar{\delta}_{k}}(x, t):=\left(1 / \lambda_{k}\right) u^{\delta_{n_{k}}}\left(\lambda_{k} x, \lambda_{k}^{2} t\right)$ satisfies $u^{\bar{\delta}_{k}} \rightarrow \alpha x_{1}^{+}$in $\{t \leqslant 0\}$, uniformly on compact subsets. Since $u^{\bar{\delta}_{k}}$ is a solution to $\left(P_{\bar{\delta}_{k}}\right)$, we may apply Theorem 5.1 and deduce that $\alpha=\alpha(M)$.

Let us finally see that $U=\alpha(M) x_{1}^{+}$in $\{t \leqslant 0\}$. In fact, $U \geqslant 0, \partial\{U>0\} \neq \varnothing$ and thus by Theorem 6.2 we have $|\nabla U| \leqslant \alpha(M)$. Using the fact that $U \equiv 0$ in $\left\{x_{1}=0\right\} \cap\{t \leqslant 0\}$ we deduce that

$$
U \leqslant \alpha(M) x_{1} \quad \text { in }\left\{x_{1}>0\right\} \cap\{t \leqslant 0\} .
$$


Consider the function $V:=\alpha(M) x_{1}-U$ which is nonnegative in $\left\{x_{1}>0\right\} \cap\{t \leqslant 0\}$ and satisfies $V=0$ on $\left\{x_{1}=0\right\} \cap\{t \leqslant 0\}$. Moreover, if we define (see Remark 3.2

$$
\tilde{\mathcal{L}} V=\operatorname{div} \tilde{F}(\nabla V)-V_{t} \quad \text { with } \quad \tilde{F}(p)=-F\left(\alpha(M) e_{1}-p\right),
$$

we see that $\tilde{\mathcal{L}} V=0$ in $\{U>0\}$.

Next, assume there exists $(\bar{x}, \bar{t}) \in\left\{x_{1}>0\right\} \cap\{t \leqslant 0\}$ such that $V(\bar{x}, \bar{t})=0$. Then $U>0$ in $Q_{\rho}^{-}(\bar{x}, \bar{t})$ for some $\rho>0$, and therefore $\tilde{\mathcal{L}} V=0$ in $Q_{\rho}^{-}(\bar{x}, \bar{t})$. It follows from the strong maximum principle (Thm. 5, Chap. 3 in [26]) that $V \equiv 0$ in $Q_{\rho}^{-}(\bar{x}, \bar{t})$. If we now define $\mathcal{R}$ to be the set of points in $\{U>0\} \cap\left\{x_{1}>0\right\} \cap\{t<\bar{t}\}$ which can be connected to $(\bar{x}, \bar{t})$ by a continuous curve in $\{U>0\}$ along which the $t$-coordinate is nondecreasing, then $\mathcal{R} \supset Q_{\rho}^{-}(\bar{x}, \bar{t})$ and $U=\alpha(M) x_{1}$ in $\mathcal{R}$ so necessarily $\mathcal{R}=\left\{x_{1}>0\right\} \cap\{t<\bar{t}\}$ and $V \equiv 0$ there.

It follows that the only possibilities are

$$
V \equiv 0 \quad \text { in }\left\{x_{1}>0\right\} \cap\{t \leqslant 0\},
$$

or else

$$
V>0 \quad \text { in }\left\{x_{1}>0\right\} \cap\{\delta<t \leqslant 0\}
$$

for some $\delta<0$. If 7.6 holds, since $\tilde{\mathcal{L}} V \leqslant 0$ and $V(0,0)=0$, Lemma 3.4 yields $V(x, t) \geqslant$ $\sigma x_{1}+o\left(|x|+|t|^{1 / 2}\right)$ in $\left\{x_{1}>0\right\} \cap\{t \leqslant 0\}$ for some $\sigma>0$. But this contradicts the fact that 7.3 . holds with $\alpha=\alpha(M)$. Therefore, we have (7.5), which completes the proof.

For a general operator we get

THEOREM 7.2 Let $u^{\varepsilon_{j}}$ be a family of nonnegative solutions to $\left(P_{\varepsilon_{j}}\right)$ in a domain $\mathcal{D} \subset \mathbb{R}^{N+1}$ such that $u^{\varepsilon_{j}} \rightarrow u$ on compact subsets of $\mathcal{D}$ and $\varepsilon_{j} \rightarrow 0$, and assume that the free boundary $\mathcal{D} \cap \partial\{u>0\}$ is a differentiable surface at $\left(x_{0}, t_{0}\right) \in \mathcal{D} \cap \partial\{u>0\}$ with nonvanishing spatial normal. Assume that $u$ is nondegenerate at $\left(x_{0}, t_{0}\right)$. Then

$$
u(x, t)=\alpha(v, M)\left\langle x-x_{0}, v\right\rangle^{+}+o\left(\left|x-x_{0}\right|+\left|t-t_{0}\right|^{1 / 2}\right),
$$

where $v$ is the inward unit spatial normal at $\left(x_{0}, t_{0}\right)$ and $\alpha(v, M)$ is given by 5.4 .

Proof. We assume that $v=e_{1}$. We consider, for $\lambda>0, u_{\lambda}(x, t):=(1 / \lambda) u\left(x_{0}+\lambda x, t_{0}+\lambda^{2} t\right)$, and a sequence $\lambda_{j} \rightarrow 0$. Then there exists a function $U \in \operatorname{Lip}(1,1 / 2)$ in $\mathbb{R}^{N+1}$ such that, for a subsequence, $u_{\lambda_{j}} \rightarrow U$ uniformly on compact subsets of $\mathbb{R}^{N+1}$.

It is not difficult to see that $U \equiv 0$ in $\left\{x_{1}<0\right\}$ and $U \geqslant 0$ in $\left\{x_{1}>0\right\}$. In particular, $U=0$ in $\left\{x_{1}=0\right\}$. Moreover, $\mathcal{L} U=0$ in $\left\{x_{1}>0\right\}$, because this holds for $u_{\lambda}$ in every compact set $K \subset \subset$ $\left\{x_{1}>0\right\}$ for $\lambda$ small enough.

Furthermore, in $\left\{x_{1}>0\right\}$, we have either $U>0$ or $U \equiv 0$. In fact, if $U(\bar{x}, \bar{t})=0$ at some point $(\bar{x}, \bar{t}) \in\left\{x_{1}>0\right\}$, then $U \equiv 0$ in $\left\{x_{1}>0\right\} \cap\{t \leqslant \bar{t}\}$ by the strong maximum principle. Then, defining $\mathcal{A}:=\left\{x_{1}>0\right\} \cap\{t>\bar{t}\}$, we see that $U \in \operatorname{Lip}(1,1 / 2)$ and $U \geqslant 0$ and $\mathcal{L} U=0$ in $\mathcal{A}$. Since, in addition, $U=0$ on $\partial_{p} \mathcal{A}$ we apply Lemma 3.3 to conclude that $U \equiv 0$ in $\mathcal{A}$ and thus in $\left\{x_{1}>0\right\}$.

Now, since $U \equiv 0$ in $\left\{x_{1}>0\right\}$ contradicts the nondegeneracy assumption, we infer $U>0$ in $\left\{x_{1}>0\right\}$.

Next, as in the proof of Theorem 7.1. we deduce that

$$
U(x, t)=\alpha\left(e_{1}, M\right) x_{1}^{+}+o\left(|x|+|t|^{1 / 2}\right) \quad \text { in }\left\{x_{1}>0\right\} \cap\{t \leqslant 0\} .
$$


In addition, in the present situation we apply Theorem 6.3 to obtain $|\nabla U| \leqslant \alpha\left(e_{1}, M\right)$ and then deduce that $U \leqslant \alpha\left(e_{1}, M\right) x_{1}$ in $\left\{x_{1}>0\right\}$.

We now define $V:=\alpha\left(e_{1}, M\right) x_{1}-U$ and $\tilde{\mathcal{L}} V=\operatorname{div} \tilde{F}(\nabla V)-V_{t}$ with $\tilde{F}(p)=$ $-F\left(\alpha\left(e_{1}, M\right) e_{1}-p\right)$. Then $\tilde{\mathcal{L}} V=0$ and $V \geqslant 0$ in $\left\{x_{1}>0\right\}$, and $V=0$ on $\left\{x_{1}=0\right\}$. Thus, an argument similar to the one applied before to $U$ allows us to deduce that in $\left\{x_{1}>0\right\}$ either $V>0$ or $V \equiv 0$.

If we had $V>0$ in $\left\{x_{1}>0\right\}$, then the application of Lemma 3.4 would contradict 7.8). Therefore, $U=\alpha\left(e_{1}, M\right) x_{1}^{+}$in $\mathbb{R}^{N+1}$, which implies the result.

\section{Viscosity solutions}

In this section we prove that the limit functions are viscosity supersolutions and viscosity subsolutions of the free boundary problem $(P)$ under suitable assumptions (Theorems 8.1 and 8.2 . We refer to Remarks 8.2 to 8.4 for a discussion of our assumptions.

The notion of viscosity solution for a general class of free boundary problems of evolution type (including the Stefan problem) was introduced in [2-4] in order to study the regularity properties of the free boundary. There, the authors used classical subsolutions and supersolutions as test functions to define viscosity solutions (see also [16] for a discussion and for an equivalent concept of viscosity solution).

In the same spirit as in [2-4], a notion of viscosity solution was introduced in [15] for the linear version of the free boundary problem $(P)$ considered in the present paper. We here generalize the definition in [15] to quasilinear problems.

We notice that the free boundary condition in the free boundary problem $(P)$ does not involve the speed of the free boundary as occurred in the class of free boundary problems considered in [2-4]. In fact, the free boundary condition in problem $(P)$ is of the type of the conditions appearing in the class of elliptic free boundary problems considered in [10-12] (see [16] for equivalent notions of viscosity solutions of such elliptic free boundary problems).

We start the section with some definitions, which extend naturally to any domain in $\mathbb{R}^{N+1}$.

Definition 8.1 Let $\tilde{Q}:=\tilde{B} \times\left(T_{1}, T_{2}\right), \tilde{B}$ a ball in $\mathbb{R}^{N}$, and let $v$ be a continuous function in $\overline{\tilde{Q}}$. Then $v$ is called a classical subsolution (resp. supersolution) to $(P)$ in $\tilde{Q}$ if

(1) $\mathcal{L} v \geqslant 0$ (resp. $\leqslant 0)$ in $\Omega^{+}:=\tilde{Q} \cap\{v>0\}$.

(2) $v$ is nonnegative, $v \in C^{2,1}\left(\overline{\Omega^{+}}\right)$.

(3) For any $(x, t) \in \partial \Omega^{+} \cap \tilde{Q}, \nabla v^{+}(x, t) \neq 0$ and

$$
v_{v}^{+} \geqslant \alpha(v, M) \quad\left(\text { resp. } v_{v}^{+} \leqslant \alpha(v, M)\right),
$$

where $v:=\nabla v^{+} /\left|\nabla v^{+}\right|$.

We say that $v$ is a classical solution to $(P)$ in $\tilde{Q}$ if it is both a classical subsolution and a classical supersolution to $(P)$ in $\tilde{Q}$.

DEFINITION 8.2 Let $u$ be a continuous, nonnegative function in $\tilde{Q}$. Then $u$ is called a viscosity subsolution (resp. supersolution) to $(P)$ in $\tilde{Q}$ if, for every subcylinder $Q \subset \subset \tilde{Q}$ and for every $v$ which is a classical supersolution (resp. subsolution) to $(P)$ in $Q$,

$$
u \leqslant v \quad(\text { resp. } u \geqslant v) \quad \text { on } \partial_{p} Q
$$


and

$$
\left.v>0 \text { on } \overline{\{u>0\}} \cap \partial_{p} Q \quad \text { (resp. } u>0 \text { on } \overline{\{v>0\}} \cap \partial_{p} Q\right)
$$

implies that $u \leqslant v($ resp. $u \geqslant v)$ in $Q$.

The function $u$ is called a viscosity solution to $(P)$ if it is both a viscosity subsolution and a viscosity supersolution to $(P)$.

REMARK 8.1 If $u$ is a classical subsolution (resp. supersolution) to $(P)$ in $\tilde{Q}$ then $u$ is a viscosity subsolution (resp. supersolution) to $(P)$ in $\tilde{Q}$. In fact, this can be seen by reasoning as in the proofs of Theorems 8.1 and 8.2 .

DEFINITION 8.3 Let $u$ be a continuous nonnegative function in a domain $\mathcal{D} \subset \mathbb{R}^{N+1}$. We say that a point $\left(x_{0}, t_{0}\right) \in \mathcal{D} \cap \partial\{u>0\}$ is a regular point from the right (resp. left) if there exist $\tau>0$ and $y_{0} \in \mathbb{R}^{N}$, with $\left|x_{0}-y_{0}\right|=\tau$, such that $\mathcal{P} \mathcal{F}_{\tau}\left(y_{0}, t_{0}\right) \subset\{u>0\}$ (resp. $\left.\mathcal{P} \mathcal{F}_{\tau}\left(y_{0}, t_{0}\right) \subset\{u \equiv 0\}^{\circ}\right)$. Here we have set $\mathcal{P} \mathcal{F}_{\tau}\left(y_{0}, t_{0}\right)=\left\{(x, t):\left|x-y_{0}\right|^{2}+\left(t_{0}-t\right)<\tau^{2}, t<t_{0}\right\}$.

We first obtain

THEOREM 8.1 Let $u^{\varepsilon_{j}}$ be nonnegative solutions to $\left(P_{\varepsilon_{j}}\right)$ in a domain $\mathcal{D} \subset \mathbb{R}^{N+1}$ such that $u^{\varepsilon_{j}} \rightarrow u$ uniformly on compact subsets of $\mathcal{D}$ and $\varepsilon_{j} \rightarrow 0$. Assume one of the following conditions is satisfied:

(1) Assumption (2.6) holds.

(2) For every point $(x, t) \in \mathcal{D} \cap \partial\{u>0\}$ which is regular from the right,

$$
\liminf _{r \rightarrow 0^{+}} \frac{\left|Q_{r}^{-}(x, t) \cap\{u \equiv 0\}\right|}{\left|Q_{r}^{-}(x, t)\right|}>0 .
$$

Then $u$ is a viscosity supersolution to $(P)$ in $\mathcal{D}$.

Proof. We will modify the proof of Theorem 4.1 in [15]. Let $Q \subset \subset \mathcal{D}$ be a cylinder which will be assumed to be $B_{1}(0) \times(0, T)$, and let $v$ be a classical subsolution to $(P)$ in $Q$ satisfying $u \geqslant v$ on $\partial_{p} Q$ and $u>0$ on $\overline{\{v>0\}} \cap \partial_{p} Q$. We will show that $u \geqslant v$ in $Q$.

If $\overline{\{v>0\}} \cap \partial_{p} Q=\emptyset$ then $v=0$ on $\partial_{p} Q$ and therefore $v \equiv 0$ and $u \geqslant v$ in $Q$.

If $\overline{\{v>0\}} \cap \partial_{p} Q \neq \varnothing$, it follows from the continuity of $u$ and $v$ that $u>0$ in $\overline{\{v>0\}} \cap Q$ for $0 \leqslant t \leqslant \bar{t}$ for some small $\bar{t}>0$. It is not hard to see that $u>0$ in $\overline{\{v>0\}} \cap Q$ for $0 \leqslant t \leqslant s$ implies $u \geqslant v$ in $Q \cap\{0 \leqslant t \leqslant s\}$. We set

$$
t_{0}=\sup \{0<s<T: u>0 \text { in } \overline{\{v>0\}} \cap Q \cap\{0 \leqslant t<s\}\},
$$

and we will get a contradiction assuming $t_{0}<T$. We have $t_{0}>0$ and $u \geqslant v$ in $Q \cap\left\{0 \leqslant t \leqslant t_{0}\right\}$. In addition, there exists a sequence $\left(x_{n}, t_{n}\right) \rightarrow\left(x_{0}, t_{0}\right) \in \bar{Q}$ such that $u\left(x_{n}, t_{n}\right)=0$ and $\left(x_{n}, t_{n}\right) \in$ $\overline{\{v>0\}} \cap Q$. Then $u\left(x_{0}, t_{0}\right)=v\left(x_{0}, t_{0}\right)=0$ and $\left(x_{0}, t_{0}\right) \in \partial\{v>0\} \cap Q$. Since $v$ is a classical subsolution to $(P)$, there exists a sequence $y_{n} \rightarrow x_{0}$ such that $0<v\left(y_{n}, t_{0}\right) \leqslant u\left(y_{n}, t_{0}\right)$, so that we have proved

$$
u \geqslant v \quad \text { in } Q \cap\left\{0 \leqslant t \leqslant t_{0}\right\}, \quad\left(x_{0}, t_{0}\right) \in \partial\{u>0\} \cap \partial\{v>0\} \cap Q .
$$

Now for $\lambda>0$ set

$$
u_{\lambda}(x, t):=\frac{1}{\lambda} u\left(x_{0}+\lambda x, t_{0}+\lambda^{2} t\right), \quad v_{\lambda}(x, t):=\frac{1}{\lambda} v\left(x_{0}+\lambda x, t_{0}+\lambda^{2} t\right) .
$$


Since $u, v \in \operatorname{Lip}(1,1 / 2)$ in $Q$ and $u_{\lambda}(0,0)=v_{\lambda}(0,0)=0$, there exist a sequence $\lambda_{n} \rightarrow 0$ and $u_{0}, v_{0} \in \operatorname{Lip}(1,1 / 2)$ in $\mathbb{R}^{N+1}$ such that $v_{\lambda_{n}} \rightarrow v_{0}$ and $u_{\lambda_{n}} \rightarrow u_{0}$ uniformly on compact subsets of $\mathbb{R}^{N+1}$. Since $v$ is a classical subsolution to $(P)$, if we assume that $\nabla v^{+}\left(x_{0}, t_{0}\right) /\left|\nabla v^{+}\left(x_{0}, t_{0}\right)\right|=e_{1}$ and we set $\bar{\alpha}=\left|\nabla v^{+}\left(x_{0}, t_{0}\right)\right|$, we see that

$$
v_{0}(x, t)=\bar{\alpha} x_{1}^{+}, \quad \bar{\alpha} \geqslant \alpha\left(e_{1}, M\right) .
$$

In addition, $u_{0}(0,0)=0$, and moreover $u_{0} \geqslant v_{0}$ when $t<0$, so that $\mathcal{L} u_{0}=0$ in $\left\{x_{1}>0, t<0\right\}$. We also notice that

$$
\operatorname{div} \tilde{F}\left(\nabla w_{0}\right)-w_{0 t}=0 \quad \text { in }\left\{x_{1}>0, t<0\right\}, \quad w_{0}:=u_{0}-v_{0}, \tilde{F}(p):=F\left(p+\bar{\alpha} e_{1}\right) .
$$

There are two possibilities depending on whether the following assertion holds or not:

There is a $\delta<0$ such that $u_{0}-v_{0}>0$ when $x_{1}>0$ and $\delta<t<0$.

Suppose that $(8.2)$ does not hold. Then there is a sequence $\left(x_{n}, t_{n}\right)$ in $\left\{x_{1}>0, t<0\right\}$ such that $t_{n} \rightarrow 0$ and $\left(u_{0}-v_{0}\right)\left(x_{n}, t_{n}\right)=0$. Making use of (8.1) and of Remark 3.2 we apply the strong maximum principle (Thm. 5, Chap. 3 in [26]) to $u_{0}-v_{0}$ to conclude

$$
u_{0} \equiv v_{0}=\bar{\alpha} x_{1}^{+} \quad \text { in }\left\{x_{1} \geqslant 0, t \leqslant 0\right\},
$$

implying that

$$
\frac{1}{\lambda_{n}}(u-v)\left(\lambda_{n} e_{1}+x_{0}, t_{0}\right) \rightarrow 0 \quad \text { as } n \rightarrow \infty .
$$

We write $(x, t)=\left(x_{1}, x^{\prime}, t\right)$ and for small $\rho, r>0$ we define

$$
E=\left\{f\left(x^{\prime}, t\right)<x_{1}<f\left(x^{\prime}, t\right)+\rho,\left|x^{\prime}-x_{0}^{\prime}\right|<r,\left|t-t_{0}\right|<r^{2}\right\},
$$

where $f$ is a $C^{2,1}$ function in a neighborhood of $\left(x_{0}^{\prime}, t_{0}\right)$ such that for a small $r_{0}>0$,

$$
B_{r_{0}}\left(x_{0}, t_{0}\right) \cap \partial\{v>0\}=B_{r_{0}}\left(x_{0}, t_{0}\right) \cap\left\{(x, t): x_{1}=f\left(x^{\prime}, t\right)\right\}
$$

and

$$
B_{r_{0}}\left(x_{0}, t_{0}\right) \cap\{v>0\}=B_{r_{0}}\left(x_{0}, t_{0}\right) \cap\left\{(x, t): x_{1}>f\left(x^{\prime}, t\right)\right\} .
$$

If $r, \rho$ are small enough, then $E \subset\{v>0\}$. Observe that $\mathcal{L} u=0$ in $E \cap\left\{t<t_{0}\right\}$ with $u \in$ $C^{2,1}\left(E \cap\left\{t<t_{0}\right\}\right)$, and $\mathcal{L} v \geqslant 0$ in $E$ with $v \in C^{2,1}(\bar{E})$. Then, in $E \cap\left\{t<t_{0}\right\}, \widehat{\mathcal{L}}(u-v) \leqslant 0$ for a linear uniformly parabolic operator $\widehat{\mathcal{L}}$. In fact, in $E \cap\left\{t<t_{0}\right\}$, we have

$$
\begin{aligned}
0 & \geqslant \sum_{i, j=1}^{N}\left(\frac{\partial F_{i}}{\partial p_{j}}(\nabla u) \partial_{x_{i} x_{j}} u-\frac{\partial F_{i}}{\partial p_{j}}(\nabla v) \partial_{x_{i} x_{j}} v\right)-\partial_{t}(u-v) \\
& =\sum_{i, j=1}^{N} a_{i j}(x, t) \partial_{x_{i} x_{j}}(u-v)+\sum_{i, j=1}^{N}\left(\frac{\partial F_{i}}{\partial p_{j}}(\nabla u)-\frac{\partial F_{i}}{\partial p_{j}}(\nabla v)\right) \partial_{x_{i} x_{j}} v-\partial_{t}(u-v),
\end{aligned}
$$

where $a_{i j}(x, t):=\frac{\partial F_{i}}{\partial p_{j}}(\nabla u)$. Now, fix $(x, t) \in E \cap\left\{t<t_{0}\right\}$ and write, for $s \in[0,1]$,

$$
h(s):=\sum_{i, j=1}^{N} \frac{\partial F_{i}}{\partial p_{j}}(\nabla v+s(\nabla u-\nabla v)) \partial_{x_{i} x_{j}} v
$$


Since $F \in C^{2}$, we have $h \in C^{1}$. Hence the second expression in the last line in (8.4) can be written as

$$
h(1)-h(0)=\int_{0}^{1} h^{\prime}(s) \mathrm{d} s=\sum_{k=1}^{N} b_{k}(x, t) \partial_{x_{k}}(u-v),
$$

with

$$
b_{k}(x, t):=\sum_{i, j=1}^{N} \int_{0}^{1} \frac{\partial^{2} F_{i}}{\partial p_{j} \partial p_{k}}(\nabla v+s(\nabla u-\nabla v)) \partial_{x_{i} x_{j}} v \mathrm{~d} s .
$$

Then, since $\nabla v, \partial_{x_{i} x_{j}} v, \nabla u \in L^{\infty}\left(E \cap\left\{t<t_{0}\right\}\right)$ and $F \in C^{2}$, we see that $a_{i j}, b_{k} \in L^{\infty}\left(E \cap\left\{t<t_{0}\right\}\right)$ and $u-v$ satisfies

$$
\widehat{\mathcal{L}}(u-v):=\sum_{i, j=1}^{N} a_{i j}(x, t) \partial_{x_{i} x_{j}}(u-v)+\sum_{k=1}^{N} b_{k}(x, t) \partial_{x_{k}}(u-v)-\partial_{t}(u-v) \leqslant 0
$$

in $E \cap\left\{t<t_{0}\right\}$. Now, the strong maximum principle implies that $u-v$ is positive in $E \cap\left\{t<t_{0}\right\}$.

We then observe that, since $(u-v)\left(x_{0}, t_{0}\right)=0$, and $\partial E=\partial\{v>0\} \in C^{2,1}$ in a neighborhood of $\left(x_{0}, t_{0}\right)$ with $\nabla v^{+}\left(x_{0}, t_{0}\right) /\left|\nabla v^{+}\left(x_{0}, t_{0}\right)\right|=e_{1}$, we can apply Lemma 2.6 in [25] at $\left(x_{0}, t_{0}\right)$ to conclude

which contradicts 8.3 .

$$
\liminf _{\lambda \rightarrow 0^{+}} \frac{(u-v)\left(\lambda e_{1}+x_{0}, t_{0}\right)}{\lambda} \geqslant C>0,
$$

Hence necessarily 8.2 holds. Recalling 8.1 and Remark 3.2 we apply Theorem A.1 to deduce that

$$
\left(u_{0}-v_{0}\right)(x, t)=\sigma x_{1}^{+}+o\left(|x|+|t|^{1 / 2}\right)
$$

when $x_{1}>0, t<0$ for some $\sigma>0$. That is,

$$
u_{0}(x, t)=\alpha x_{1}^{+}+o\left(|x|+|t|^{1 / 2}\right) \quad \text { in }\left\{x_{1}>0, t<0\right\} \text { with } \alpha>\bar{\alpha} .
$$

Now for $\lambda>0$ set

$$
\left(u_{0}\right)_{\lambda}(x, t)=\frac{1}{\lambda} u_{0}\left(\lambda x, \lambda^{2} t\right) .
$$

There exist a sequence $\bar{\lambda}_{n} \rightarrow 0$ and $u_{00} \in \operatorname{Lip}(1,1 / 2)$ in $\mathbb{R}^{N+1}$ such that $\left(u_{0}\right)_{\bar{\lambda}_{n}} \rightarrow u_{00}$ uniformly on compact subsets of $\mathbb{R}^{N+1}$. We have

$$
u_{00}(x, t)=\alpha x_{1}^{+} \quad \text { in }\left\{x_{1}>0, t<0\right\} .
$$

We now apply Corollary A.1 to $u_{00}$ in $\left\{x_{1}<0, t<0\right\}$ to get

$$
u_{00}(x, t)=\tilde{\alpha} x_{1}^{-}+o\left(|x|+|t|^{1 / 2}\right) \quad \text { in }\left\{x_{1}<0, t<0\right\}
$$

for some $\tilde{\alpha} \geqslant 0$. We consider

$$
\left(u_{00}\right)_{\lambda}(x, t)=\frac{1}{\lambda} u_{00}\left(\lambda x, \lambda^{2} t\right) .
$$

There is a sequence $\tilde{\lambda}_{n} \rightarrow 0$ and $u_{000} \in \operatorname{Lip}(1,1 / 2)$ in $\mathbb{R}^{N+1}$ such that $\left(u_{00}\right)_{\tilde{\lambda}_{n}} \rightarrow u_{000}$ uniformly on compact subsets of $\mathbb{R}^{N+1}$, and moreover

$$
u_{000}(x, t)=\alpha x_{1}^{+}+\tilde{\alpha} x_{1}^{-} \quad \text { for } t \leqslant 0 .
$$


Applying Lemma 4.3 three times we find a sequence $\varepsilon_{j}^{000} \rightarrow 0$ and solutions $u_{j}^{\varepsilon_{j}^{000}}$ to $\left(P_{\varepsilon_{j}^{000}}\right)$ in $Q_{1}(0,0)$ such that $\frac{u^{\varepsilon_{j}}}{u_{0}} \rightarrow u_{000}$ uniformly on compact subsets of $Q_{1}(0,0)$.

In case 2.6 holds, we apply either Theorem 5.1 or Theorem 5.2 to $u_{000}$ in a neighborhood of some point $(0, \bar{t})$ with $\bar{t}<0$. Now assume 2.6 does not hold. Since $\left(x_{0}, t_{0}\right)$ is a regular point from the right,

$$
\liminf _{r \rightarrow 0^{+}} \frac{\left|Q_{r}^{-}\left(x_{0}, t_{0}\right) \cap\{u \equiv 0\}\right|}{\left|Q_{r}^{-}\left(x_{0}, t_{0}\right)\right|}>0 .
$$

This implies that $\tilde{\alpha}=0$, and thus we can apply Theorem 5.1. In any case we deduce that

$$
\alpha \leqslant \alpha\left(e_{1}, M\right) \text {. }
$$

But we have $\alpha>\bar{\alpha} \geqslant \alpha\left(e_{1}, M\right)$, which is a contradiction.

Finally, we get

THEOREM 8.2 Let $u^{\varepsilon_{j}}$ be nonnegative solutions to $\left(P_{\varepsilon_{j}}\right)$ in a domain $\mathcal{D} \subset \mathbb{R}^{N+1}$ such that $u^{\varepsilon_{j}} \rightarrow u$ uniformly on compact subsets of $\mathcal{D}$ and $\varepsilon_{j} \rightarrow 0$. Assume that $u$ is nondegenerate at every regular point from the left in $\mathcal{D} \cap \partial\{u>0\}$. Then $u$ is a viscosity subsolution to $(P)$ in $\mathcal{D}$.

Proof. We will modify the proof of Theorem 4.2 in [15]. Let $Q \subset \subset \mathcal{D}$ be a cylinder which will be assumed to be $B_{1}(0) \times(0, T)$, and let $v$ be a classical supersolution to $(P)$ in $Q$ satisfying $v \geqslant u$ on $\partial_{p} Q$ and $v>0$ on $\overline{\{u>0\}} \cap \partial_{p} Q$. We will show that $v \geqslant u$ in $Q$.

If $\overline{\{u>0\}} \cap \partial_{p} Q=\emptyset$ the result follows easily. If not, we proceed as in Theorem 8.1 we define

$$
t_{0}=\sup \{0<s<T: v>0 \text { in } \overline{\{u>0\}} \cap Q \cap\{0 \leqslant t<s\}\},
$$

and we will get a contradiction, assuming $t_{0}<T$.

From the definition of $t_{0}$, it follows that $t_{0}>0$ and $v \geqslant u$ in $Q \cap\left\{0 \leqslant t \leqslant t_{0}\right\}$. In addition, there exists a sequence $\left(x_{n}, t_{n}\right) \rightarrow\left(x_{0}, t_{0}\right) \in \bar{Q}$ such that $v\left(x_{n}, t_{n}\right)=0$ and $\left(x_{n}, t_{n}\right) \in \overline{\{u>0\}} \cap Q$. Clearly, $u\left(x_{0}, t_{0}\right)=v\left(x_{0}, t_{0}\right)=0$ and $\left(x_{0}, t_{0}\right) \in \partial\{u>0\} \cap Q$. If $\left(x_{0}, t_{0}\right) \in\{v \equiv 0\}^{\circ}$ then, for $\delta$ small, we have $u=v=0$ in $B_{\delta}\left(x_{0}, t_{0}\right) \cap\left\{t<t_{0}\right\}$, which contradicts our hypothesis. Thus,

$$
v \geqslant u \quad \text { in } Q \cap\left\{0 \leqslant t \leqslant t_{0}\right\}, \quad\left(x_{0}, t_{0}\right) \in \partial\{u>0\} \cap \partial\{v>0\} \cap Q .
$$

We consider $u_{\lambda}, v_{\lambda}$ as in Theorem 8.1 . There exist a sequence $\lambda_{n} \rightarrow 0$ and $u_{0}, v_{0}$ such that $v_{\lambda_{n}} \rightarrow v_{0}$ and $u_{\lambda_{n}} \rightarrow u_{0}$ uniformly on compact subsets of $\mathbb{R}^{N+1}$. We have

$$
v_{0}(x, t)=\bar{\alpha} x_{1}^{+}, \quad 0<\bar{\alpha} \leqslant \alpha\left(e_{1}, M\right)
$$

where $\bar{\alpha}=\left|\nabla v^{+}\left(x_{0}, t_{0}\right)\right|$ and we have assumed that $\nabla v^{+}\left(x_{0}, t_{0}\right) /\left|\nabla v^{+}\left(x_{0}, t_{0}\right)\right|=e_{1}$.

In addition, $u_{0}(0,0)=0$ and $v_{0} \geqslant u_{0}$ when $t \leqslant 0$, and thus

$$
u_{0}(x, t) \equiv 0 \quad \text { in }\left\{x_{1} \leqslant 0, t \leqslant 0\right\} .
$$

We also notice that

$$
\begin{aligned}
& \tilde{\mathcal{L}} w_{0} \leqslant 0 \quad \text { in }\left\{x_{1}>0, t<0\right\}, \quad \tilde{\mathcal{L}} w_{0}=0 \quad \text { in }\left\{u_{0}>0\right\}, \quad \text { where } \\
& w_{0}:=v_{0}-u_{0}, \quad \tilde{\mathcal{L}} w_{0}:=\operatorname{div} \tilde{F}\left(\nabla w_{0}\right)-w_{0 t}, \quad \tilde{F}(p):=-F\left(\bar{\alpha} e_{1}-p\right) .
\end{aligned}
$$


In a similar way to Theorem 8.1, we consider two cases depending on whether the following assertion holds or not:

$$
\text { There is a } \delta<0 \text { such that } v_{0}-u_{0}>0 \text { when } x_{1}>0 \text { and } \delta<t<0 \text {. }
$$

Suppose 8.7 does not hold. This implies that there is a sequence $\left(x_{n}, t_{n}\right)$ in $\left\{x_{1}>0, t<0\right\}$ such that $t_{n} \rightarrow 0$ and $\left(v_{0}-u_{0}\right)\left(x_{n}, t_{n}\right)=0$. Making use of 8.6 and of Remark 3.2 and reasoning as we did in Theorem 7.1 (with $V$ ), we conclude that $w_{0} \equiv 0$ in $\left\{x_{1}>0\right\} \cap\left\{t<t_{n}\right\}$, and therefore

$$
u_{0} \equiv v_{0}=\bar{\alpha} x_{1}^{+} \quad \text { in }\left\{x_{1} \geqslant 0, t \leqslant 0\right\},
$$

implying that

$$
\frac{1}{\lambda_{n}}(v-u)\left(x_{0}+\lambda_{n} e_{1}, t_{0}\right) \rightarrow 0 \quad \text { as } n \rightarrow \infty .
$$

As in Theorem 8.1 we define, for small $\rho, r>0$,

$$
E=\left\{f\left(x^{\prime}, t\right)<x_{1}<f\left(x^{\prime}, t\right)+\rho,\left|x^{\prime}-x_{0}^{\prime}\right|<r,\left|t-t_{0}\right|<r^{2}\right\},
$$

where $f$ is a $C^{2,1}$ function in a neighborhood of $\left(x_{0}^{\prime}, t_{0}\right)$ defining $\partial\{v>0\}$ in a neighborhood of $\left(x_{0}, t_{0}\right)$ as in Theorem 8.1. If $r, \rho$ are small enough, then $E \subset\{v>0\}$.

We know that $v-u \geqslant 0$ in $E \cap\left\{t<t_{0}\right\}$. Let us show that we actually have $v-u>0$ in $E \cap\left\{t \leqslant t_{0}\right\}$. In fact, in $\{u>0\} \cap\left\{t<t_{0}\right\}, \mathcal{L} u=0$ with $u \in C^{2,1}$ and we have $\mathcal{L} v \leqslant 0$ in $E$ with $v \in C^{2,1}(\bar{E})$. Then we can argue as in 8.4 in the set $\{u>0\} \cap\left\{t<t_{0}\right\}$ to conclude that $\widehat{\mathcal{L}}(v-u) \leqslant 0$ in $\{u>0\} \cap\left\{t<t_{0}\right\}$ for the same linear uniformly parabolic operator $\widehat{\mathcal{L}}$ with bounded coefficients defined in 8.5 . Then the strong maximum principle implies that $v-u>0$ in $\{u>0\} \cap\left\{t \leqslant t_{0}\right\}$, and thus $v-u>0$ in $E \cap\left\{t \leqslant t_{0}\right\}$.

On the other hand, we can argue as in $(8.4)$ to deduce that $\widehat{\mathcal{L}}^{\varepsilon_{j}}\left(v-u^{\varepsilon_{j}}\right) \leqslant 0$ in $E \cap\left\{t<t_{0}\right\}$ for the linear uniformly parabolic operator

$$
\widehat{\mathcal{L}}^{\varepsilon_{j}} U:=\sum_{i, l=1}^{N} a_{i l}^{\varepsilon_{j}}(x, t) \partial_{x_{i} x_{l}} U+\sum_{k=1}^{N} b_{k}^{\varepsilon_{j}}(x, t) \partial_{x_{k}} U-\partial_{t} U .
$$

Here

$$
a_{i l}^{\varepsilon_{j}}(x, t):=\frac{\partial F_{i}}{\partial p_{l}}\left(\nabla u^{\varepsilon_{j}}\right), \quad b_{k}^{\varepsilon_{j}}(x, t):=\sum_{i, l=1}^{N} \int_{0}^{1} \frac{\partial^{2} F_{i}}{\partial p_{l} \partial p_{k}}\left(\nabla v+s\left(\nabla u^{\varepsilon_{j}}-\nabla v\right)\right) \partial_{x_{i} x_{l}} v \mathrm{~d} s
$$

and so $a_{i l}^{\varepsilon_{j}}, b_{k}^{\varepsilon_{j}} \in L^{\infty}\left(E \cap\left\{t<t_{0}\right\}\right)$, with bounds independent of $j$.

We now define $c^{\varepsilon_{j}}:=\sup _{E \cap\left\{t<t_{0}\right\}}\left|u^{\varepsilon_{j}}-u\right|$ and $w^{\varepsilon_{j}}=v-u^{\varepsilon_{j}}+c^{\varepsilon_{j}}$. Then $\widehat{\mathcal{L}}^{\varepsilon_{j}} w^{\varepsilon_{j}} \leqslant 0$ in $E \cap\left\{t<t_{0}\right\}$. In addition, from the discussion above we see that $w^{\varepsilon_{j}} \geqslant 0$ in $E \cap\left\{t<t_{0}\right\}$ and moreover, for every $K \subset \subset E$, there exists $c_{K}>0$ such that $w^{\varepsilon_{j}}>c_{K}$ in $K \cap\left\{t \leqslant t_{0}\right\}$.

Since, in a neighborhood of $\left(x_{0}, t_{0}\right), \partial E=\partial\{v>0\} \in C^{2,1}$ with $\nabla v^{+}\left(x_{0}, t_{0}\right) /\left|\nabla v^{+}\left(x_{0}, t_{0}\right)\right|$ $=e_{1}$, there exists $\tau>0$ such that

$$
\mathcal{P F}:=\left\{(x, t):\left|x-y_{0}\right|^{2}+\left(t_{0}-t\right)<\tau^{2}, t<t_{0}\right\} \subset E \cap\left\{t<t_{0}\right\}
$$

with $y_{0}=x_{0}+\tau e_{1}$. Defining

$$
\mathcal{P}:=\left\{(x, t) \in \mathcal{P F}:\left|x-y_{0}\right|>\tau / 2\right\},
$$


we can follow the ideas in Lemma 2.6 in [25] and construct a smooth function $w$ in $\overline{\mathcal{P}}$ such that

$$
\widehat{\mathcal{L}}^{\varepsilon_{j}} w \geqslant 0 \quad \text { in } \mathcal{P}, \quad w \leqslant w^{\varepsilon_{j}} \quad \text { on } \partial_{p} \mathcal{P}, \quad \text { with } w\left(x_{0}, t_{0}\right)=0, w_{x_{1}}\left(x_{0}, t_{0}\right)>0 .
$$

We point out that we can take the function $w$ independent of $j$, since the constants in the construction can be chosen independent of $j$. We then have $v-u^{\varepsilon_{j}}+c^{\varepsilon_{j}} \geqslant w$ in $\overline{\mathcal{P}}$, and thus

$$
v-u \geqslant w \text { in } \overline{\mathcal{P}},
$$

implying

$$
\liminf _{\lambda \rightarrow 0^{+}} \frac{1}{\lambda}(v-u)\left(x_{0}+\lambda e_{1}, t_{0}\right) \geqslant w_{x_{1}}\left(x_{0}, t_{0}\right)>0,
$$

which contradicts 8.8 .

The above argument implies that necessarily 8.7 holds. Then, recalling $(8.6)$ and Remark 3.2 , we can apply Theorem A.1 to $w_{0}$ to obtain

$$
\left(v_{0}-u_{0}\right)(x, t)=\sigma x_{1}^{+}+o\left(|x|+|t|^{1 / 2}\right)
$$

when $x_{1} \geqslant 0, t<0$ for some $\sigma>0$. That is, we have proved that

$$
u_{0}(x, t)=\alpha x_{1}^{+}+o\left(|x|+|t|^{1 / 2}\right) \quad \text { in }\{t<0\} \text { with } 0 \leqslant \alpha<\bar{\alpha} .
$$

Now consider $\left(u_{0}\right)_{\lambda}$ for $\lambda>0$. There is a sequence $\bar{\lambda}_{n} \rightarrow 0$ and $u_{00} \in \operatorname{Lip}(1,1 / 2)$ in $\mathbb{R}^{N+1}$ such that $\left(u_{0}\right)_{\bar{\lambda}_{n}} \rightarrow u_{00}$ uniformly on compact subsets of $\mathbb{R}^{N+1}$. We have

$$
u_{00}(x, t)=\alpha x_{1}^{+} \quad \text { in }\{t \leqslant 0\}
$$

with $\bar{\alpha}>\alpha \geqslant 0$. Since $\left(x_{0}, t_{0}\right)$ is a regular point from the left, $u$ is nondegenerate at $\left(x_{0}, t_{0}\right)$, and therefore $\alpha>0$.

Applying Lemma 4.3 twice we find a sequence $\varepsilon_{j}^{00} \rightarrow 0$ and solutions $u^{\varepsilon_{j}^{00}}$ to $\left(P_{\varepsilon_{j}^{00}}\right)$ in $Q_{1}(0,0)$ such that $u^{\varepsilon_{j}^{00}} \rightarrow u_{00}$ uniformly on compact subsets of $Q_{1}(0,0)$. We now apply Theorem 5.1 to $u_{00}$ in a neighborhood of some point $(0, \bar{t})$ with $\bar{t}<0$ and deduce that $\alpha=\alpha\left(e_{1}, M\right)$. Since $\alpha<\bar{\alpha} \leqslant \alpha\left(e_{1}, M\right)$, we get a contradiction, thus proving the theorem.

REMARK 8.2 We point out that limit functions with regular points from the left that are degenerate do exist. This can be seen, for instance, with the following example. For simplicity we consider the heat operator.

Let $\mathcal{O} \subset \mathbb{R}^{N}$ be a smooth bounded domain, $T>0$, and let $u$ be smooth in $\overline{\mathcal{O}} \times[0, T]$ such that

$$
\begin{aligned}
\Delta u-\partial_{t} u=0 & \text { in } \mathcal{O} \times(0, T), \\
u=0 & \text { in } \overline{\mathcal{O}} \times\left[0, t_{0}\right], \\
u>0 & \text { in } \overline{\mathcal{O}} \times\left(t_{0}, T\right],
\end{aligned}
$$

for some $0<t_{0}<T$. Set $u^{\varepsilon}=u+\varepsilon$ for $\varepsilon>0$, and $\mathcal{D}=\mathcal{O} \times(0, T)$. Then $u=\lim _{\varepsilon \rightarrow 0} u^{\varepsilon}$, with $u^{\varepsilon}$ nonnegative solutions to

$$
\Delta u^{\varepsilon}-\partial_{t} u^{\varepsilon}=\beta_{\varepsilon}\left(u^{\varepsilon}\right) \quad \text { in } \mathcal{D} \text {. }
$$

We now observe that $\mathcal{D} \cap \partial\{u>0\}=\mathcal{O} \times\left\{t_{0}\right\}$. Moreover, for any $x_{0} \in \mathcal{O}$, we see that $\left(x_{0}, t_{0}\right)$ is a regular point from the left, and it is a degenerate free boundary point, in the sense that (7.2) in Definition 7.2 is not satisfied. 
REMARK 8.3 Let us now present an example of a limit function having regular points from the left that are degenerate, for which the conclusion of Theorem 8.2 does not hold. For simplicity we consider the heat operator, and we let $N=1$ and $M=\int \beta=1 / 2$.

In $Q=(-1,1) \times(0,3 / 4)$ define

$$
v(x, t)=\chi_{\{x+t>1\}}(\exp (x+t-1)-1)+\chi_{\{-x+t>1\}}(\exp (-x+t-1)-1)
$$

(i.e., colliding traveling waves before singularity develops, see Figure 1 in [28]).

Then $v$ is a classical supersolution to $(P)$ in $Q$ according to Definition 8.1 . Observe that

$$
v \geqslant e^{1 / 4}-1=c_{0}>0 \quad \text { on } \partial_{p} Q \cap\{t \geqslant 1 / 4\} .
$$

Now let $u$ be a solution of the heat equation in some bounded domain $\mathcal{D} \subset \mathbb{R}^{2}$, with $Q \subset \subset \mathcal{D}$, such that

$$
u=0 \quad \text { on } \mathcal{D} \cap\{t \leqslant 1 / 4\}, \quad 0<u<c_{0} \quad \text { on } \mathcal{D} \cap\{t>1 / 4\}
$$

( $u$ is a limit function of nonnegative solutions to problems $\left(P_{\varepsilon}\right)$ chosen as in Remark 8.2).

Then, by construction, $v \geqslant u$ on $\partial_{p} Q$ and $v>0$ on $\overline{\{u>0\}} \cap \partial_{p} Q$. But it is not true that $v \geqslant u$ in $Q$, since $u(0, t)>0=v(0, t)$ for $t>1 / 4$.

REMARK 8.4 Our assumptions in Theorem 8.2 exclude regular points from the left that are degenerate. In fact, we want to avoid situations like the one in Remark 8.3 where the result fails to hold. However, our assumptions in Theorem 8.2 do not exclude free boundary points with other types of singular behavior-points that can certainly be present in limit functions. For an example of a free boundary point with another type of singular behavior see, for instance, Section 1 in [17], where a self-similar limit function with an extinction point is constructed for the heat operator.

REMARK 8.5 In the recent work [1], under a certain flatness condition on the free boundary in some space direction, the free boundary was proved to be smooth in the case of the heat operator. In particular, Corollary 8.5 in [1] says that, in the case of the heat operator, if (7.7) holds at $\left(x_{0}, t_{0}\right) \in$ $\mathcal{D} \cap \partial\{u>0\}$ (and thus $u$ is nondegenerate at $\left(x_{0}, t_{0}\right)$ ), then the free boundary is a smooth surface in a neighborhood of $\left(x_{0}, t_{0}\right)$.

\section{Appendix A}

In this section we obtain results on asymptotic developments for nonnegative functions $U$ satisfying either $\mathcal{L} U \leqslant 0$ or $\mathcal{L} U \geqslant 0$, which are used throughout the paper. These results are modifications of Lemma A1 and Corollary A1 in [14].

For $v \in \mathbb{S}^{N-1}$ and $\tau>0$, we define

$$
\mathcal{C}_{\tau}^{v}:=Q_{\tau}^{-}(0,0) \cap\{\langle x, v\rangle>0\},
$$

and we first prove

Theorem A.1 Let $U \in \operatorname{Lip}(1,1 / 2)$ in $\overline{\mathcal{C}_{\tau}^{v}}$ with $U_{t} \in L_{\text {loc }}^{2}\left(\mathcal{C}_{\tau}^{\nu}\right)$ for some $\tau>0$. Assume that $U>0$ and $\mathcal{L} U \leqslant 0$ in $\mathcal{C}_{\tau}^{\nu}$, and that $U(0,0)=0$. Then, in $\mathcal{C}_{\tau}^{\nu}, U$ has the asymptotic development

$$
U(x, t)=\alpha\langle x, v\rangle+o\left(|x|+|t|^{1 / 2}\right)
$$

with some $\alpha>0$. 
Proof. We assume $\tau>1$ and $v=e_{1}$ and we write $\mathcal{C}_{\tau}:=\mathcal{C}_{\tau}^{e_{1}}$. Let

$$
\varepsilon_{k}:=\sup \left\{\varepsilon: U(x, t) \geqslant \varepsilon x_{1} \text { in } \mathcal{C}_{2^{-k}}\right\} .
$$

Then $\varepsilon_{k}$ is a nondecreasing sequence and it is bounded from above by the Lipschitz seminorm of $U$ in the space variables. Let $\alpha:=\lim _{k \rightarrow \infty} \varepsilon_{k}$. Then $U(x, t) \geqslant \alpha x_{1}+o\left(|x|+|t|^{1 / 2}\right)$ in $\mathcal{C}_{1}$. Assume that there is a sequence $\left(x^{k}, t^{k}\right) \in \mathcal{C}_{1}$ with $r_{k}:=\max \left\{\left|x^{k}\right|,\left|t^{k}\right|^{1 / 2}\right\} \rightarrow 0$ such that

$$
U\left(x^{k}, t^{k}\right)-\alpha x_{1}^{k} \geqslant \delta_{0} r_{k}
$$

for some $\delta_{0}>0$ and let

$$
U_{k}(x, t):=\frac{U\left(r_{k} x, r_{k}^{2} t\right)}{r_{k}} .
$$

Since $U \in \operatorname{Lip}(1,1 / 2)$ it follows that there exists a nonnegative function $V \in \operatorname{Lip}(1,1 / 2)$ in $\overline{\mathcal{C}}_{1}$ such that, for a subsequence, $U_{k} \rightarrow V$ uniformly in $\overline{\mathcal{C}}_{1}$. From the construction we have $V-\alpha x_{1} \geqslant 0$ in $\overline{\mathcal{C}_{1}}$ and, in addition, there is a point $(\bar{x}, \bar{t}) \in \partial_{p} \mathcal{C}_{1} \cap\left\{x_{1}>0\right\}$ and $\varepsilon>0$ such that

$$
V-\alpha x_{1} \geqslant \delta_{0} / 2 \quad \text { on } \partial_{p} \mathcal{C}_{1} \cap B_{\varepsilon}(\bar{x}, \bar{t}) .
$$

We can take $\varepsilon$ small enough so that $\overline{B_{\varepsilon}(\bar{x}, \bar{t})} \subset\left\{x_{1}>0\right\}$.

Recalling Remark 3.2 we define $\tilde{F}(p):=F\left(p+\alpha e_{1}\right)$ and let $w$ be a solution of

$$
\tilde{\mathcal{L}} w:=\operatorname{div} \tilde{F}(\nabla w)-w_{t}=0 \quad \text { in } \mathcal{C}_{1},
$$

with smooth boundary data such that

$$
\begin{aligned}
w=0 & \text { on } \partial_{p} \mathcal{C}_{1} \backslash B_{\varepsilon / 2}(\bar{x}, \bar{t}), \\
w=\delta_{0} / 2 & \text { on } \partial_{p} \mathcal{C}_{1} \cap B_{\varepsilon / 4}(\bar{x}, \bar{t}), \\
0 \leqslant w \leqslant \delta_{0} / 2 & \text { on } \partial_{p} \mathcal{C}_{1} \cap B_{\varepsilon / 2}(\bar{x}, \bar{t}) .
\end{aligned}
$$

Then $w \in C^{2,1}\left(\mathcal{C}_{1}\right) \cap C\left(\overline{\mathcal{C}}_{1}\right), w>0$ in $\mathcal{C}_{\sigma}$ for some $\sigma>0$, and $w(0,0)=0$, and therefore from Lemma 3.4 it follows that

$$
w(x, t) \geqslant \mu x_{1} \quad \text { in } \mathcal{C}_{\gamma}
$$

for some small $\mu, \gamma>0$. Letting

$$
c_{k}:=\sup _{\mathcal{C}_{1}}\left|V-U_{k}\right| \quad \text { and } \quad \tilde{U}_{k}:=U_{k}-\alpha x_{1},
$$

and observing that $\tilde{\mathcal{L}} \tilde{U}_{k} \leqslant 0$, we deduce from Lemma 3.2 that $\tilde{U}_{k} \geqslant w-c_{k}$ in $\mathcal{C}_{1}$, and thus

$$
\tilde{U}_{k} \geqslant \mu x_{1}-c_{k} \text { in } \overline{\mathcal{C}}_{\gamma} .
$$

We also notice that

$$
\tilde{U}_{k} \geqslant 0 \quad \text { on }\left\{x_{1}=0\right\} .
$$

Now let $\Omega$ be a smooth domain such that $B_{3 \gamma / 4}(0) \cap\left\{x_{1}>0\right\} \subset \Omega \subset B_{\gamma}(0) \cap\left\{x_{1}>0\right\}$ (and thus $B_{3 \gamma / 4}(0) \cap\left\{x_{1}=0\right\} \subset \partial \Omega$ ), and let $\mathcal{D}_{\gamma}:=\Omega \times\left(-\gamma^{2}, 0\right]$ (notice that $\mathcal{C}_{3 \gamma / 4} \subset \mathcal{D}_{\gamma} \subset \mathcal{C}_{\gamma}$ ). 
Next, let $w_{k} \in C^{2,1}\left(\overline{\mathcal{D}}_{\gamma}\right)$ be such that $\tilde{\mathcal{L}} w_{k}=0$ in $\mathcal{D}_{\gamma}$ and $w_{k}=\psi\left(\mu x_{1}-c_{k}\right)$ on $\partial_{p} \mathcal{D}_{\gamma}$, with $\psi$ a smooth function satisfying

$$
\psi \equiv 0 \quad \text { in } Q_{\gamma / 4}(0,0), \quad \psi \equiv 1 \quad \text { in } \mathbb{R}^{N+1} \backslash Q_{\gamma / 2}(0,0), \quad 0 \leqslant \psi \leqslant 1 .
$$

By combining A.1 and A.2 it follows that $\tilde{U}_{k} \geqslant w_{k}$ on $\partial_{p} \mathcal{D}_{\gamma}$. Then we apply Lemma 3.2 to infer that $\tilde{U}_{k} \geqslant w_{k}$ in $\mathcal{D}_{\gamma}$.

Moreover, $w_{k} \rightarrow \mu x_{1}$ uniformly in $\overline{\mathcal{D}}_{\gamma}$, and

$$
\nabla w_{k} \rightarrow \mu e_{1} \quad \text { uniformly in } \overline{\mathcal{D}}_{\gamma} .
$$

This implies that, in $\mathcal{C}_{\gamma / 4}$,

$$
U_{k}(x, t)-\alpha x_{1}=\tilde{U}_{k}(x, t) \geqslant w_{k}(x, t) \geqslant \frac{\mu}{2} x_{1}
$$

if $k$ is large enough. This is in contradiction with the definition of $\alpha$ and hence

$$
U(x, t)=\alpha x_{1}+o\left(|x|+|t|^{1 / 2}\right) \quad \text { in } \mathcal{C}_{1} .
$$

Since $U>0$ in $\mathcal{C}_{\tau}$ and $\tau>1$, we apply Lemma 3.4 to deduce that $\alpha>0$, thus concluding the proof.

As a consequence we obtain

COROLlaRY A.1 Let $U \in \operatorname{Lip}(1,1 / 2)$ be nonnegative in $\overline{\mathcal{C}_{\tau}^{v}}$ with $U_{t} \in L_{\text {loc }}^{2}\left(\mathcal{C}_{\tau}^{v}\right)$ for some $\tau>0$. Assume that $\mathcal{L} U \geqslant 0$ in $\mathcal{C}_{\tau}^{v}$ and that $U \equiv 0$ on $\{\langle x, v\rangle=0\}$. Then, in $\mathcal{C}_{\tau}^{\nu}, U$ has the asymptotic development

with some $\beta \geqslant 0$.

$$
U(x, t)=\beta\langle x, v\rangle+o\left(|x|+|t|^{1 / 2}\right)
$$

Proof. Let $V(x, t):=2 L\langle x, \nu\rangle-U(x, t)$ where $L$ is the Lipschitz constant of $U$ with respect to the space variables. Defining $\tilde{F}(p)=-F(2 L v-p)$, we observe that $\tilde{\mathcal{L}} V:=\operatorname{div} \tilde{F}(\nabla V)-\partial_{t} V \leqslant 0$ in $\mathcal{C}_{\tau}^{v}$. Together with Remark 3.2, this implies that $V$ satisfies the assumptions of Theorem A.1

\section{Appendix B}

For the sake of completeness we devote this appendix to briefly review how equation (1.1) appears in combustion theory and derive equation $\left(P_{\varepsilon}\right)$ as well. For more details we refer to the classical literature on the subject [6, 7, 8]. See also [27].

Let us first recall how equation (1.1) appears in the description of the propagation of curved premixed equi-diffusional deflagration flames with high activation energy. In fact, we consider a situation in which we have a homogeneous mixture of one fuel and one oxidizer. The model under consideration is based on isobaric and constant-density approximations, in which pressure and density fluctuations are neglected so that the reaction-diffusion equations for the combustion variables (temperature and mass fraction of the reactant) decouple from the equations involving the hydrodynamic variables (density, pressure and velocity). We thus obtain a system for the temperature $T$ and the mass fraction of the reactant $Y$ of the form

$$
\left\{\begin{array}{c}
\partial_{t} T+\mathrm{v} \cdot \nabla T-\operatorname{div}\left(D_{T} \nabla T\right)=Y f(T), \\
\partial_{t} Y+\mathrm{v} \cdot \nabla Y-\operatorname{div}\left(D_{Y} \nabla Y\right)=-Y f(T),
\end{array}\right.
$$


where v is a specified velocity field and $D_{T}, D_{Y}$ are positive constants ( $D_{T}$ is the thermal diffusivity and $D_{Y}$ the mass diffusivity).

We next consider $\mathrm{v}=0$ together with the hypothesis of equi-diffusion (i.e., $D_{T}=D_{Y}$ ). Then a suitable change of variables allows us to assume that $D_{T}=D_{Y}=1$ and system (B.1) becomes

$$
\left\{\begin{array}{l}
\partial_{t} T-\Delta T=Y f(T) \\
\partial_{t} Y-\Delta Y=-Y f(T)
\end{array}\right.
$$

We introduce the function $H=T+Y$, which by (B.2) satisfies $\Delta H-\partial_{t} H=0$. Assuming that we work in the whole space and that $H(x, 0)=1$, we obtain $H \equiv 1$ and therefore we get the equation

$$
\Delta u-\partial_{t} u=\beta(u),
$$

for $u:=Y=1-T$, with $\beta(s)=s f(1-s)$.

The function $f$ involves a constant $E$ called the activation energy. In the present situation, the limit analysis of flame propagation for very large $E$ can be done by considering (B.3) with a family of reaction functions $\beta_{\varepsilon}$, chosen as in Section 1] and then letting $\varepsilon \rightarrow 0$ (see [27]).

A natural extension to the above model consists in allowing nonlinear diffusion (cf. [27, Sect. 15]). In fact, it is natural in the theories of thermal propagation and combustion to consider nonlinearities of quasilinear type-where different nonlinearities reflect properties of the particular medium under consideration (see for instance [9] and the survey paper [20], as well as their references; see also [21]).

Thus, we propose to study the above model replacing in $\mathrm{B} .1$ the linear laws $D_{T} \nabla T$ and $D_{Y} \nabla Y$ by more general nonlinear ones of the form $F_{T}(\nabla T)$ and $F_{Y}(\nabla Y)$. We then obtain the system

$$
\left\{\begin{array}{l}
\partial_{t} T+\mathrm{v} \cdot \nabla T-\operatorname{div}\left(F_{T}(\nabla T)\right)=Y f(T) \\
\partial_{t} Y+\mathrm{v} \cdot \nabla Y-\operatorname{div}\left(F_{Y}(\nabla Y)\right)=-Y f(T) .
\end{array}\right.
$$

We assume again $\mathrm{v}=0$ and a single diffusion law (i.e., $F_{T}=F_{Y}=F$ ) and we get

$$
\left\{\begin{array}{l}
\partial_{t} T-\operatorname{div}(F(\nabla T))=Y f(T), \\
\partial_{t} Y-\operatorname{div}(F(\nabla Y))=-Y f(T) .
\end{array}\right.
$$

Considering as before the function $H=T+Y$ and assuming that $H(x, 0)=1$ and $F(-p)=$ $-F(p)$, we find again that $H \equiv 1$, since now, by (B.4), $H$ satisfies an equation of the type $\sum_{i, j=1} a_{i j}(x, t) \partial_{x_{i} x_{j}} H+\sum_{k=1} b_{k}(x, t) \partial_{x_{k}} H-\partial_{t} H=0$ (here we use arguments similar to those in $(8.5)$.

Thus, the equation for $u:=Y=1-T$ of interest in the high activation energy analysis now reads

$$
\operatorname{div} F(\nabla u)-\partial_{t} u=\beta_{\varepsilon}(u)
$$

with $\beta_{\varepsilon}$ as in Section 1

\section{Acknowledgements}

The authors want to thank the referees for their helpful comments that improved the presentation of the paper. This work has been partially supported by the ALFA-Project AML/B7-311/97/0666/II0143-FCD "Partial Differential Equations in Industry and Engineering" funded by the European 
Union. The research of the first author has been partially supported by Fundación Antorchas Project 13900-5, ANPCyT PICT No. 03-13719, Universidad de Buenos Aires grant X052 and CONICET PIP 5478. The first author is a member of CONICET. The second author has been partially supported by the Austrian Science Fund (FWF) through the Wissenschaftskolleg "Differential Equations" and through the Wittgenstein 2000 award of Peter Markowich.

\section{REFERENCES}

1. Andersson, J., \& Weiss, G. S. A parabolic free boundary problem with Bernoulli type condition on the free boundary. Preprint (arXiv:math/0608746v1[math.AP]).

2. Athanasopoulos, I., Caffarelli, L. A., \& Salsa, S. Caloric functions in Lipschitz domains and the regularity of solutions to phase transition problems. Ann. of Math. 143 (1996), 413-434. Zbl 0853.35049 MR 1394964

3. Athanasopoulos, I., Caffarelli, L. A., \& Salsa, S. Regularity of the free boundary in parabolic phase transition problems. Acta Math. 176 (1996), 245-282. Zbl 0891.35164 MR 1397563

4. Athanasopoulos, I., Caffarelli, L. A., \& Salsa, S. Phase transition problems of parabolic type: Flat free boundaries are smooth. Comm. Pure Appl. Math. 51 (1998), 77-112. Zbl 0924.35197 MR 1486632

5. Berestycki, H., Caffarelli, L. A., \& Nirenberg, L. Uniform estimates for regularization of free boundary problems. Analysis and Partial Differential Equations, C. Sadosky (ed.), Lecture Notes in Pure Appl. Math. 122, Dekker, New York (1990), 567-619. Zbl 0702.35252 MR 1044809

6. Buckmaster, J. D. An introduction to combustion theory. The Mathematics of Combustion, J. D. Buckmaster (ed.), SIAM, Philadelphia (1985), 3-46.

7. Buckmaster, J. D., \& Ludford, G. S. S. Theory of Laminar Flames. Cambridge Univ. Press, Cambridge (1982). Zbl 0557.76001 MR 0666866

8. Buckmaster, J. D., \& LUdFORD, G. S. S. Lectures on Mathematical Combustion. CBMS-NSF Reg. Conf. Ser. Appl. Math. 43, SIAM, Philadelphia (1983). Zbl 0574.76001 MR 0765073

9. Budd, C., \& GAlaktionov, V. Stability and spectra of blow up in problems with quasi-linear gradient diffusivity. Proc. Roy. Soc. London Ser. A 454 (1998), 2371-2407. Zbl 1023.35053 MR 1653356

10. CAfFarelli, L. A. A Harnack inequality approach to the regularity of free boundaries. Part I, Lipschitz free boundaries are $C^{1, \alpha}$. Rev. Mat. Iberoamer. 3 (1987), 139-162. Zbl 0676.35085 MR 0990856

11. CAfFarelli, L. A. A Harnack inequality approach to the regularity of free boundaries. Part II, Flat free boundaries are Lipschitz. Comm. Pure Appl. Math. 42 (1989), 55-78. Zbl 0676.35086 MR 0973745

12. Caffarelli, L. A. A Harnack inequality approach to the regularity of free boundaries. Part III, Existence theory, compactness and dependence on X. Ann. Scuola Norm. Sup. Pisa 15 (1988), 583-602. Zbl 0702.35249 MR 1029856

13. CAFFARELli, L. A. Uniform Lipschitz regularity of a singular perturbation problem. Differential Integral Equations 8 (1995), 1585-1590. Zbl 0863.35008 MR 1347971

14. Caffarelli, L. A., Lederman, C., \& Wolanski, N. Uniform estimates and limits for a two phase parabolic singular perturbation problem. Indiana Univ. Math. J. 46 (1997), 453-489. Zbl 0909.35012 MR 1481599

15. Caffarelli, L. A., Lederman, C., \& Wolanski, N. Pointwise and viscosity solutions for the limit of a two phase parabolic singular perturbation problem. Indiana Univ. Math. J. 46 (1997), 719-740. Zbl 0909.35013 MR 1488334

16. Caffarelli, L. A., \& Salsa, S. A Geometric Approach to Free Boundary Problems. Grad. Stud. Math. 68, Amer. Math. Soc., Providence, RI (2005). Zbl 1083.35001 MR 2145284

17. Caffarelli, L. A., \& VÁzquez, J. L. A free boundary problem for the heat equation arising in flame propagation. Trans. Amer. Math. Soc. 347 (1995), 411-441. Zbl 0814.35149 1260199 
18. FERnÁndeZ Bonder, J., \& WOLANSKI, N. A free boundary problem in combustion theory. Interfaces Free Bound. 2 (2000), 381-411. Zbl 0969.35143 MR 1789173

19. Friedman, A. Partial Differential Equations of Parabolic Type. Prentice-Hall, Englewood Cliffs, NJ (1964). Zbl 0144.34903 MR 0181836

20. Galaktionov, V. A., \& VÁzQuez, J. L. The problem of blow-up in nonlinear parabolic equations. Discr. Cont. Dynam. Systems 8 (2002), 399-433. Zbl 1010.35057 MR 1897690

21. Gusachenko, L. K. Burning of fuels with nonisotropic heat conduction. Combustion, Explosion and Shock Waves 3 (1969), 193-194.

22. LAdyŽEnsKaja, O. A., Solonnikov, V. A., \& UrAL'CeVA, N. N. Linear and Quasilinear Equations of Parabolic Type. Transl. Math. Monogr. 23, Amer. Math. Soc., Providence, RI (1967). Zbl 0174.15403 MR 0241822

23. Lederman, C., \& WolAnski, N. Viscosity solutions and regularity of the free boundary for the limit of an elliptic two phase singular perturbation problem. Ann. Scuola Norm. Sup. Pisa 27 (1998), 253-288. Zbl 0931.35200 MR 1664689

24. Lederman, C., \& Wolanski, N. Singular perturbation in a nonlocal diffusion problem. Comm. Partial Differential Equations 31 (2006), 195-241. Z Zbl 1100.35126 MR 2209752

25. Lieberman, G. M. Second Order Parabolic Differential Equations. World Sci., River Edge, NJ (1996). Zbl 0884.35001 MR 1465184

26. Protter, M. H., \& Weinberger, H. F. Maximum Principles in Differential Equations. Prentice-Hall, Englewood Cliffs, NJ (1967). Zbl 0153.13602 MR 0219861

27. VÁzquez, J. L. The free boundary problem for the heat equation with fixed gradient condition. Free Boundary Problems, Theory and Applications (Zakopane, 1995), Pitman Res. Notes Math. Ser. 363, M. Niezgódka and P. Strzelecki (eds.), Longman, Harlow (1996), 277-302. Zbl 0867.35120 MR 1462990

28. WeISS, G. S. A singular limit arising in combustion theory: fine properties of the free boundary. Calc. Var. Partial Differential Equations 17 (2003), 311-340. Zbl 1045.35114 MR 1989835

29. Zeldovich, YA. B., \& FrAnK-KAMEnEtSKi, D. A. The theory of thermal propagation of flames. $Z h$. Fiz. Khim. 12 (1938), 100-105 (in Russian); English transl. in: Collected Works of Ya. B. Zeldovich, Vol. 1, Princeton Univ. Press, 1992. 